\title{
ICE AND DUST IN THE QUIESCENT MEDIUM OF ISOLATED DENSE CORES*
}

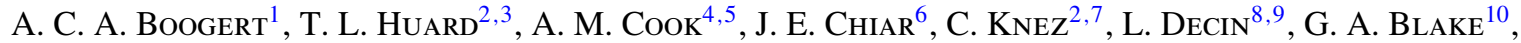 \\ A. G. G. M. TIELEnS ${ }^{11}$, AND E. F. VAN DishoeCK ${ }^{11,12}$ \\ ${ }^{1}$ IPAC, NASA Herschel Science Center, Mail Code 100-22, California Institute of Technology, Pasadena, CA 91125, USA; aboogert@ipac.caltech.edu \\ ${ }^{2}$ Department of Astronomy, University of Maryland, College Park, MD 20742, USA \\ ${ }^{3}$ Columbia Astrophysics Laboratory, Columbia University, New York, NY 10027, USA \\ ${ }^{4}$ New York Center for Astrobiology and Department of Physics, Applied Physics \& Astronomy, Rensselaer Polytechnic Institute, \\ 110 Eighth Street, Troy, NY 12180, USA \\ ${ }^{5}$ IPAC, Mail Code 100-22, California Institute of Technology, Pasadena, CA 91125, USA \\ ${ }^{6}$ SETI Institute, Carl Sagan Center, 189 Bernardo Av., Mountain View, CA 94043, USA \\ 7 Johns Hopkins University Applied Physics Laboratory, 11100 Johns Hopkins Road, Laurel, MD 20723, USA \\ ${ }^{8}$ Instituut voor Sterrenkunde, Katholieke Universiteit Leuven, Celestijnenlaan 200D, 3001 Leuven, Belgium \\ ${ }^{9}$ Sterrenkundig Instituut Anton Pannekoek, University of Amsterdam, Science Park 904, NL-1098 Amsterdam, The Netherlands \\ ${ }^{10}$ Division of GPS, Mail Code 150-21, California Institute of Technology, Pasadena, CA 91125, USA \\ ${ }^{11}$ Leiden Observatory, Leiden University, P.O. Box 9513, 2300 RA Leiden, The Netherlands \\ ${ }^{12}$ Max Planck Institut für Extraterrestrische Physik (MPE), Giessenbachstr.1, 85748 Garching, Germany \\ Received 2010 September 15; accepted 2011 January 6; published 2011 February 14
}

\begin{abstract}
The relation between ices in the envelopes and disks surrounding young stellar objects (YSOs) and those in the quiescent interstellar medium (ISM) is investigated. For a sample of 31 stars behind isolated dense cores, ground-based and Spitzer spectra and photometry in the 1-25 $\mu \mathrm{m}$ wavelength range are combined. The baseline for the broad and overlapping ice features is modeled, using calculated spectra of giants, $\mathrm{H}_{2} \mathrm{O}$ ice and silicates. The adopted extinction curve is derived empirically. Its high resolution allows for the separation of continuum and feature extinction. The extinction between 13 and $25 \mu \mathrm{m}$ is $\sim 50 \%$ relative to that at $2.2 \mu \mathrm{m}$. The strengths of the 6.0 and $6.85 \mu \mathrm{m}$ absorption bands are in line with those of YSOs. Thus, their carriers, which, besides $\mathrm{H}_{2} \mathrm{O}$ and $\mathrm{CH}_{3} \mathrm{OH}$, may include $\mathrm{NH}_{4}^{+}, \mathrm{HCOOH}, \mathrm{H}_{2} \mathrm{CO}$, and $\mathrm{NH}_{3}$, are readily formed in the dense core phase, before stars form. The $3.53 \mu \mathrm{m} \mathrm{C}-\mathrm{H}$ stretching mode of solid $\mathrm{CH}_{3} \mathrm{OH}$ was discovered. The $\mathrm{CH}_{3} \mathrm{OH} / \mathrm{H}_{2} \mathrm{O}$ abundance ratios of $5 \%-12 \%$ are larger than upper limits in the Taurus molecular cloud. The initial ice composition, before star formation occurs, therefore depends on the environment. Signs of thermal and energetic processing that were found toward some YSOs are absent in the ices toward background stars. Finally, the peak optical depth of the $9.7 \mu \mathrm{m}$ band of silicates relative to the continuum extinction at $2.2 \mu \mathrm{m}$ is significantly shallower than in the diffuse ISM. This extends the results of Chiar et al. to a larger sample and higher extinctions.
\end{abstract}

Key words: astrochemistry - infrared: ISM - infrared: stars - ISM: abundances - ISM: molecules - stars: formation

Online-only material: color figures

\section{INTRODUCTION}

Planets and comets are assembled from the gas, dust, and ices in circumstellar disks that in turn evolve from the envelopes and clouds surrounding young stellar objects (YSOs). Many key questions about the molecular composition of protostellar environments are still unanswered. The infrared spectra of YSOs are rich in absorption features caused by the vibrational transitions of silicates and of solid $\mathrm{H}_{2} \mathrm{O}, \mathrm{CO},{ }^{13} \mathrm{CO}, \mathrm{CH}_{3} \mathrm{OH}, \mathrm{NH}_{3}$, OCS, $\mathrm{CH}_{4}, \mathrm{CO}_{2},{ }^{13} \mathrm{CO}_{2}$, and possibly of $\mathrm{OCN}^{-}, \mathrm{NH}_{4}^{+}, \mathrm{HCOOH}$, and $\mathrm{HCOO}^{-}$(Gibb et al. 2004; Boogert \& Ehrenfreund 2004; Boogert et al. 2008; Öberg et al. 2008; Zasowski et al. 2009; Bottinelli et al. 2010). Band profile studies revealed that both CO and $\mathrm{CO}_{2}$ are present in multiple components of mixed or pure ices that are formed or destroyed at different stages of the cloud and envelope evolution (Tielens et al. 1991; Gerakines et al. 1999; Boogert et al. 2002; Pontoppidan et al. 2003b, 2008). It was also shown that the prominent 6.0 and $6.85 \mu \mathrm{m}$ absorption features toward low- and high-mass YSOs are caused by the

\footnotetext{
* Some of the data presented herein were obtained at the W. M. Keck Observatory, which is operated as a scientific partnership among the California Institute of Technology, the University of California, and the National Aeronautics and Space Administration. The Observatory was made possible by the generous financial support of the W. M. Keck Foundation.
}

overlapping modes of simple species: $\mathrm{H}_{2} \mathrm{O}, \mathrm{H}_{2} \mathrm{CO}, \mathrm{HCOOH}$, $\mathrm{NH}_{3}, \mathrm{NH}_{4}^{+}$, and $\mathrm{CH}_{3} \mathrm{OH}$ (Keane et al. 2001; Boogert et al. 2008). Not all absorption observed in this wavelength region is explained, however, such as the very broad component labeled "C5" in Boogert et al. (2008). Ices processed by heat, UV photons, or cosmic-ray hits are potential candidates. Both ions (Schutte \& Khanna 2003) and hydrocarbons (Gibb \& Whittet 2002) have absorption features that are spread over much of the 5-8 $\mu \mathrm{m}$ region. Polycyclic aromatic hydrocarbon molecules embedded in the ices could be responsible for some of the absorption as well.

These molecules are formed by a complex interplay between numerous formation and destruction processes. Grain surface chemistry likely dominates the formation of molecules (e.g., $\mathrm{H}_{2} \mathrm{O}, \mathrm{CH}_{4}, \mathrm{CO}_{2}$ ) in cold, dense molecular clouds, leading to icy mantles (e.g., Tielens \& Hagen 1982). In the protostellar environment these ices may sublimate through the influence of shocks or photons in the inner envelopes and disk surfaces. The formation of complex molecules in the ices may be triggered by heating and by the absorption of energetic photons (Schutte et al. 1993; Greenberg et al. 1995) originating in the protostar-disk boundary layer. In the most shielded areas (outer envelopes, disk mid-planes), deeply penetrating cosmic rays can induce similar effects. It is still quite uncertain which 
molecule formation and destruction processes are important in these different environments. What is the composition of ices in pristine clouds, prior to star formation? To what degree does the solar system molecular inventory resemble quiescent cloud inventories?

A rigorous observational approach is required to further constrain the origin of unidentified absorption bands, especially in the 5-8 $\mu \mathrm{m}$ region, and to disentangle the many effects of the physical environment on molecular evolution. An established technique is to contrast the molecular composition toward YSOs with that toward background stars tracing quiescent molecular cloud material (e.g., Whittet et al. 1983, 1998). Large abundances of the most volatile species (e.g., pure, "apolar" $\mathrm{CO}$ ice) are observed in quiescent lines of sight, which is attributed to the absence of nearby heating sources. Likewise, amorphous ice structures are observed in these sight lines, whereas toward YSOs they are sometimes crystalline (Smith et al. 1989; Gerakines et al. 1999; Pontoppidan et al. 2008). Tight upper limits have been set to the abundance of the species $\mathrm{OCN}^{-}$(cyanate; Whittet et al. 2001) and $\mathrm{CH}_{3} \mathrm{OH}$ (Chiar et al. 1995) toward background stars, suggesting that specific physical conditions found only around YSOs may be needed to initiate their formation.

So far, mid-infrared $(\lambda>5 \mu \mathrm{m})$ studies have focused on only a few background stars: four toward the Taurus molecular cloud, one toward Serpens, and one toward IC 5146. Knez et al. (2005) show that the absorption bands in the 5-8 $\mu \mathrm{m}$ wavelength region are similar to those toward YSOs. The $15 \mu \mathrm{m} \mathrm{CO} 2$ band shows a non-polar ice component consistent with the pristine nature of the Taurus cloud (Bergin et al. 2005), a conclusion that can be drawn for Serpens and IC 5146 as well (Whittet et al. 2009).

The studied samples are too small to fully assess the ice inventory prior to star formation, given the wide range of environmental conditions possible. Much larger samples of background stars are needed, covering wider ranges of extinctions and cloud types. Selecting these samples is now possible using the catalogs produced by wide-field Spitzer IRAC and MIPS mapping projects, in combination with the Two Micron All Sky Survey (2MASS; Skrutskie et al. 2006). In this work, background stars are selected from the catalogs of nearby $(<350 \mathrm{pc})$, isolated, compact $\left(<5\right.$ arcmin), dense $\left(>10^{4} \mathrm{~cm}^{-3}\right)$ cores of the Spitzer Legacy team "From Molecular Cores to Planet Forming Disks" (c2d; Evans et al. 2003). These cores were originally identified in optically selected regions of extinction by observing transitions of $\mathrm{NH}_{3}$ sensitive to high densities $\left(\geqslant 10^{3} \mathrm{~cm}^{-3}\right.$; Myers \& Benson 1983). Based on IRAS colors, a subdivision was made between starless cores and cores containing stars, and it was concluded that roughly half the cores contain stars (Beichman et al. 1986). With the sensitive Spitzer MIPS and IRAC cameras, deep searches revealed new YSOs, down to stellar masses of $0.01 M_{\odot}$, even in some cores that were previously categorized as "starless" (Young et al. 2004).

Studying the interstellar medium (ISM) in isolated cores is attractive, because it lacks the environmental influences of outflows and the resulting turbulence often thought to dominate in regions of clustered star formation (e.g., $\rho$ Oph, Serpens). Their star formation time scales can be significantly larger due to the dominance of magnetic fields over turbulence and the resulting slow process of ambipolar diffusion (Shu et al. 1987; Evans 1999). The longer time scales may alter the gas composition, such as reduced $\mathrm{C} / \mathrm{CO}$ and $\mathrm{H} / \mathrm{H}_{2}$ ratios, which would qualitatively alter the grain surface chemistry. Furthermore, the longer exposure time of the ices to cosmic rays or cosmic-ray-induced ultraviolet photons may initiate reactions with large energy barriers leading to the formation of complex species (Greenberg et al. 1995; Bernstein et al. 2002).

While ices in the quiescent medium toward isolated dense cores have rarely been studied, the $9.7 \mu \mathrm{m}$ band of silicates received more attention. Chiar et al. (2007) found that toward the IC 5146, B68 and B59 cores, as well as some lines of sight through the Serpens and Taurus molecular clouds, the peak optical depth of the $9.7 \mu \mathrm{m}$ band relative to the $K$-band extinction is significantly shallower compared to the diffuse medium. Here, this relation will be revisited with a much larger sample. With a simultaneous analysis of the ices bands, some possible explanations of this relation can be addressed, e.g., the effects of grain growth.

Also, in the process of disentangling ice and dust features from the attenuated stellar spectra, an extinction curve needs to be assumed. Broadband and spectroscopic studies have shown that the extinction remains high even at $25 \mu \mathrm{m}$ (Chapman et al. 2009; McClure 2009). In this work, this result will be reassessed, using Spitzer spectra and source-specific models. In the process a high-resolution extinction curve will be produced, in which absorption features and continuum extinction can be separated.

Finally, this study of isolated dense cores is part of a larger program to investigate dust and ices in a range of environments. While here many cores are observed with a few background stars per core, Chiar et al. (2011) study many sight lines behind one specific core in the IC 5146 region in much detail. Efforts are under way to study large nearby clouds as well.

The selection of the background stars is described in Section 2, and the reduction of the ground-based and Spitzer spectra in Section 3. In Section 4.1, the procedure to fit the stellar continua is presented. This is a crucial step in the analysis. For this, a "high-resolution" extinction curve is needed in which ice and silicate features can be separated from continuum extinction. This extinction curve is derived in Section 4.2. Subsequently, in Section 4.3, the peak and integrated optical depths of the ice and dust features are derived, as well as column densities for the known identifications. Then in Section 4.4, the derived parameters are correlated with each other and with previously studied YSOs. In Section 5.1, the comparison of ice abundances in the background stars and YSOs is discussed, followed by a discussion on observational tracers of ice processing in Section 5.2. Section 5.3 shows how the observations of the ices further constrain explanations for the deviating extinction curve and $A_{\mathrm{K}}$ versus $\tau_{9.7}$ relation in dense clouds. Finally, the conclusions are summarized and an outlook to future studies is presented in Section 6.

\section{SOURCE SELECTION}

Background stars were selected from a sample of 69 isolated molecular cloud cores that were mapped with Spitzer/IRAC and MIPS by the c2d Legacy team (Evans et al. 2003, 2007). The cores are limited by size $(<5$ arcmin), distance $(<350 \mathrm{pc})$, and density $\left(>10^{4} \mathrm{~cm}^{-3}\right)$, and both historically designated starless and star-forming cores (from IRAS colors; Beichman et al. 1986) are included. The catalogs generated by the c2d team were systematically searched for sources meeting the following criteria.

1. The overall spectral energy distribution (SED; 2MASS 1-2 $\mu \mathrm{m}$, IRAC 3-8 $\mu \mathrm{m}$, MIPS $24 \mu \mathrm{m})$ must be that of a reddened Rayleigh-Jeans curve. 
2. Regions of high extinction $\left(A_{V} \geqslant 5 \mathrm{mag}\right)$, associated with the core, must be traced. The regional extinction was measured using the Near-Infrared Color Excess method (e.g., Lada et al. 1999), which uses five 2MASS sources nearest each target to derive statistically reliable extinctions.

3. Fluxes must be high enough ( $>5 \mathrm{mJy}$ at $8.0 \mu \mathrm{m}$ or $>30 \mathrm{mJy}$ at $15 \mu \mathrm{m}$ ) to obtain Spitzer/Infrared Spectrograph (IRS) spectra of high quality $(\mathrm{S} / \mathrm{N}>100)$ within $\sim 30$ minutes of observing time per module. This is needed to detect the often weak ice absorption features and determine their shapes and peak positions.

Thus, a sample of 32 candidate background stars was selected. One of those (2MASS J17111631-2725144 behind B59) will not be analyzed here, as its IRS spectrum shows a silicate emission band and no ice bands. The remaining 31 sources (Table 1) span a range of $A_{\mathrm{K}}$ values and are located behind 16 isolated cores. Eight out of sixteen cores are "starless" in the IRAS definition, although some of those have Spitzerdetected YSO candidates (e.g., L1014; Young et al. 2004). The selected background stars are usually within $\sim 60^{\prime \prime}$ of these YSO candidates. Spitzer spectra of many YSO candidates were obtained, but they will be presented in a forthcoming paper.

Finally, to compare the analysis methods presented here with previous work (Knez et al. 2005), two well-studied stars behind the Taurus and Serpens molecular clouds were included in the sample: 2MASS J04393886+2611266 (Elia 3-16) and 2MASS J18300061+0115201 (CK 2, [EC92] 118).

\section{OBSERVATIONS AND DATA REDUCTION}

Spitzer/IRS spectra of background stars toward isolated dense cores were obtained as part of a dedicated Open Time program (PID 20604). The complementary Taurus and Serpens background stars were observed in the c2d program (PID 172). Table 1 lists all sources with their AOR keys, and the IRS modules they were observed in. The spectra were extracted and calibrated from the two-dimensional Basic Calibrated Data produced by the standard Spitzer pipeline (version S15.3.0), using exactly the same method and routines discussed in Boogert et al. (2008). Uncertainties $(1 \sigma)$ for each spectral point were calculated using the "func" frames provided by the Spitzer pipeline.

The Spitzer spectra were complemented by ground-based Keck/NIRSPEC (McLean et al. 1998) $\mathrm{H}$-, $\mathrm{K}$-, and $\mathrm{L}$-band spectra at a resolving power of $R=2000$. These were reduced in a way standard for ground-based long-slit spectra, using bright, nearby main-sequence stars as telluric and photometric standards. In the end, all spectra were multiplied along the flux scale in order to match near-infrared broadband photometry from 2MASS (Skrutskie et al. 2006) as well as Spitzer IRAC and MIPS photometry from the c2d catalogs (Evans et al. 2007), using the appropriate filter profiles. The same photometry is used in the continuum determination discussed in Section 4. Catalog flags were taken into account, such that the photometry of sources listed as being confused within a $2^{\prime \prime}$ radius or being located within $2^{\prime \prime}$ of a mosaic edge were treated as upper limits. The c2d catalogs do not include flags for saturation. Therefore, photometry exceeding the IRAC saturation limits (at the appropriate integration times) were flagged as lower limits. Finally, since the 2MASS and Spitzer photometry represent different surveys, and their relative calibration is very important for this work, the uncertainties in the Spitzer photometry were increased by adding the zero-point magnitude uncertainties listed in Table 21 of Evans et al. (2007).
Table 1

Source Sample

\begin{tabular}{|c|c|c|c|c|}
\hline $\begin{array}{l}\text { Source } \\
\text { 2MASS J }\end{array}$ & Core $^{a}$ & AOR Key ${ }^{b}$ & Module & $\begin{array}{l}\lambda_{\mathrm{NIR}}{ }^{\mathrm{d}} \\
(\mu \mathrm{m})\end{array}$ \\
\hline $04215402+1530299$ & IRAM 04191* & 0014900992 & SL & $2.82-4.14$ \\
\hline $08052135-3909304$ & BHR 16 & 0014896128 & SL, LL2 & $\ldots$ \\
\hline $08093135-3604035$ & CG $30-31 *$ & 0014898432 & SL, LL2 & $\ldots$ \\
\hline $08093468-3605266$ & CG $30-31 *$ & 0014898176 & SL & $\ldots$ \\
\hline $12014301-6508422$ & DC $297.7-2.8^{*}$ & 0014907136 & SL, LL2 & $\ldots$ \\
\hline $12014598-6508586$ & DC 297.7-2.8* & 0014907392 & SL, LL2 & $\ldots$ \\
\hline $15421547-5248146$ & DC $3272+18$ & 0014899456 & SL & $\ldots$ \\
\hline $15421699-5247439$ & DC $3272+18$ & $0014899968^{\mathrm{e}}$ & SL & $\ldots$ \\
\hline $17111501-2726180$ & В $59 *$ & 0014900480 & SL & $2.41-4.14$ \\
\hline $17111538-2727144$ & В $59 *$ & 001 & SL & $2.41-4.14$ \\
\hline $17112005-2727131$ & B $59 *$ & 001 & SL & $2.38-4.14$ \\
\hline $17155573-2055312$ & L $100^{*}$ & 1760 & SL & $2.38-4.14$ \\
\hline $467-2057072$ & L $100 *$ & & SL & $2.39-4.14$ \\
\hline $60-2058142$ & L $100 *$ & & SL & $2.38-4.14$ \\
\hline $18140712-0708413$ & L 438 & 001 & SL & $2.82-4.14$ \\
\hline $18160600-0225539$ & CB 130-3 & 0014 & SL & $2.82-4.14$ \\
\hline $18165296-1801287$ & L 328 & 28 & SL & $2.41-4.14$ \\
\hline $18165917-1801158$ & L 328 & 07648 & SL, LL2 ${ }^{g}$ & $2.41-4.14$ \\
\hline $18170426-1802408$ & L 328 & 7904 & SL, LL2 ${ }^{g}$ & $2.06-4.14$ \\
\hline $18170429-1802540$ & & & SL, LL2 & $2.39-4.09$ \\
\hline $18170470-0814495$ & & & & $2.38-4.14$ \\
\hline $18170957-0814136$ & L 429-C & 898944 & SL, LL2 & $2.38-4.14$ \\
\hline $18171181-0814012$ & L 429-C & 0014908416 & $\mathrm{SL}$ & $2.06-4.14$ \\
\hline $18171366-0813188$ & L 429-C & 0014908672 & SL & $2.06-4.14$ \\
\hline $18172690-0438406$ & L $483^{*}$ & 0014905600 & SL, LL2 & $2.38-4.14$ \\
\hline $19201597+1135146$ & CB $188^{*}$ & 0014897408 & SL & $2.41-4.14$ \\
\hline $19201622+1136292$ & CB $188^{*}$ & 0014897664 & SL, LL2 & $2.06-4.14$ \\
\hline $19214480+1121203$ & L 673-7 & 0014904576 & SL & $2.06-4.14$ \\
\hline $21240517+4959100$ & L 1014 & 0014902528 & SL, LL2 & $1.49-4.14$ \\
\hline $21240614+4958310$ & L 1014 & 0014909696 & SL & $1.49-4.14$ \\
\hline $22063773+5904520$ & L $1165^{*}$ & 0014903040 & SL & $1.49-4.14$ \\
\hline $04393886+2611266^{\mathrm{h}}$ & Taurus MC & 0005637632 & SL, SH & $2.59-5.31^{\mathrm{i}}$ \\
\hline $18300061+0115201^{\mathrm{j}}$ & Serpens MC & 0011828224 & $\mathrm{SL}, \mathrm{SH}$ & $2.82-4.14$ \\
\hline
\end{tabular}

Notes.

a Star-forming cores as defined by IRAS studies are indicated with a "*."

b Identification number for Spitzer observations.

c Spitzer/IRS modules used: SL, short-low (5-14 $\mu \mathrm{m}, R \sim 100)$; LL2, long-low 2 (14-21.3 $\mu \mathrm{m}, R \sim 100)$; and SH, short-high (10-20 $\mu \mathrm{m}, R \sim 600)$. $\mathrm{d}$ Wavelength coverage of complementary near-infrared ground-based observations, excluding the ranges $\sim 1.79-2.06$ and $\sim 2.55-2.82 \mu \mathrm{m}$ blocked by Earth's atmosphere.

e Averaged with AOR key 0014899712

f Also observed in AOR key 0014896896, but rejected due to high solar activity. g LL2 observed, but not included in the analysis because of source confusion.

h Well-studied background star Elia 3-16 (Knez et al. 2005 and references therein).

i Near-infrared data obtained from the Infrared Space Observatory archive (Whittet et al. 1998).

j Well-studied background star [EC92] 118 (Knez et al. 2005 and references therein).

\section{RESULTS}

The observed spectra (left panels of Figure 1) show many distinct absorption features on top of reddened stellar continua: $3.0,6.0,6.8,9.7$, and $15 \mu \mathrm{m}$. These are attributed to ices and silicates, and were previously observed toward many YSOs (e.g., Boogert et al. 2008; Pontoppidan et al. 2008) and some background stars (e.g., Knez et al. 2005; Bergin et al. 2005). At a lower level, weak features from the stellar atmosphere are present as well (e.g., 2.4 and $8.0 \mu \mathrm{m}$ ). The separation of interstellar and photospheric features is essential for this work and is discussed next. 

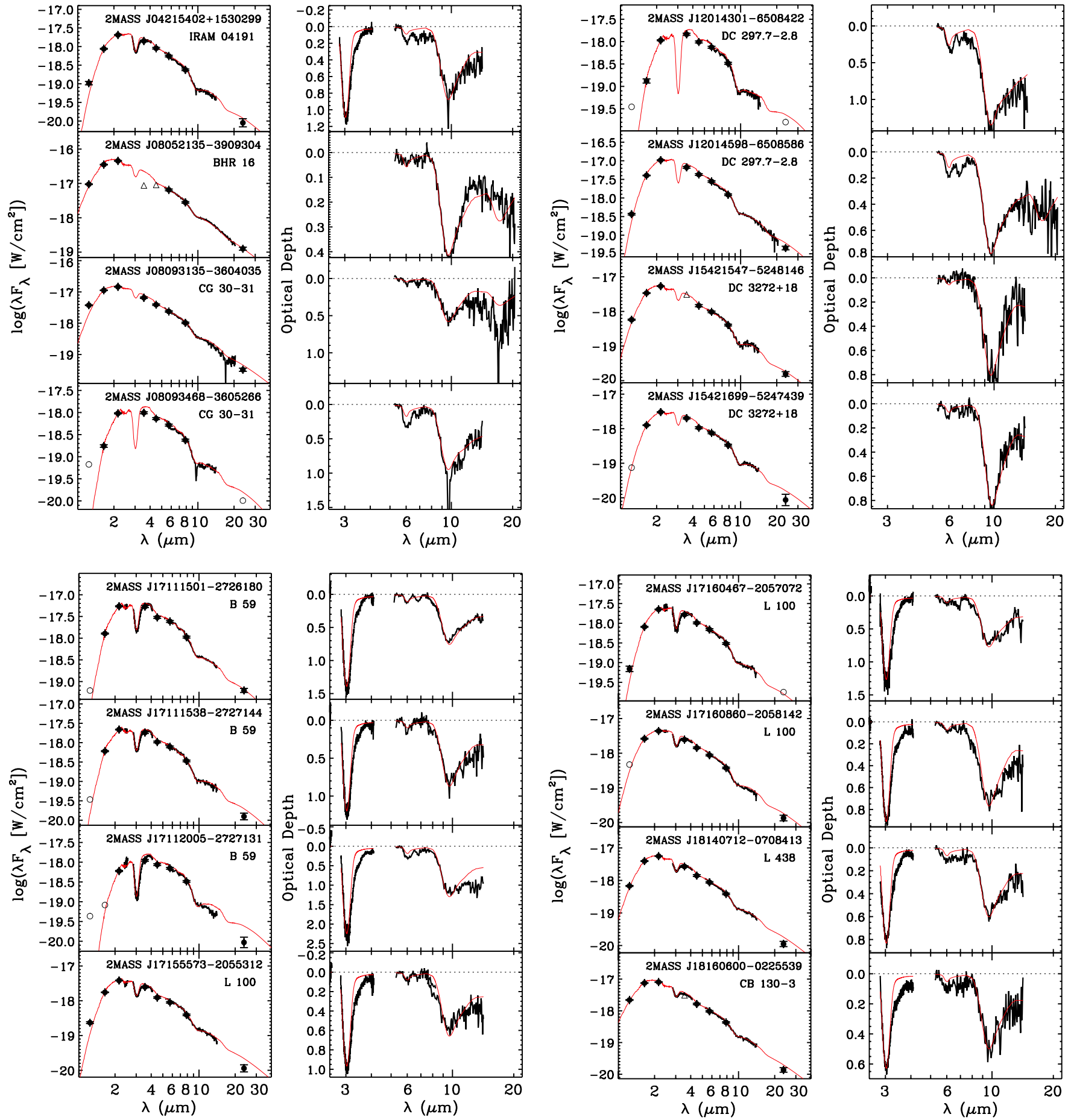

Figure 1. Left panels: observed ground-based and Spitzer/IRS spectra combined with broadband photometry (filled circles), and lower limits (open triangles) and $3 \sigma$ upper limits (open circles) thereof. The red line represents the fitted model (Section 4.1). The sources are sorted in increasing right ascension. The name of the background stars and the cores they trace are indicated. Right panels: optical depth spectra, derived using the continuum models of the left panels, excluding the modeled ice and silicate features. Note the large variation of the relative depths of the 3.0 and $9.7 \mu \mathrm{m}$ bands. The red line indicates the modeled $\mathrm{H}_{2} \mathrm{O}$ ice and silicates spectrum. For clarity, error bars of the spectral data points are not shown.

(A color version of this figure is available in the online journal.)

\subsection{Continuum Determination}

The continua and some of the absorption features in the background star spectra were fitted by minimizing the reduced$\chi^{2}$ of a model consisting of the following parameters.

1. Spectral type. An accurate spectral type determination is necessary to separate interstellar features and features from the stellar atmosphere, as well as to know the overall SED. Correction of the stellar $\mathrm{CO}$ and $\mathrm{SiO}$ bands at 5.3 and $8.0 \mu \mathrm{m}$ is particularly important, as they blend with the $6.0 \mu \mathrm{m}$ ice band and the $9.7 \mu \mathrm{m}$ band of silicates. One-dimensional, opacity-sampled spectra were calculated using the MARCS 2 model atmospheres following Decin et al. (2004). Only giants (luminosity class III) were 

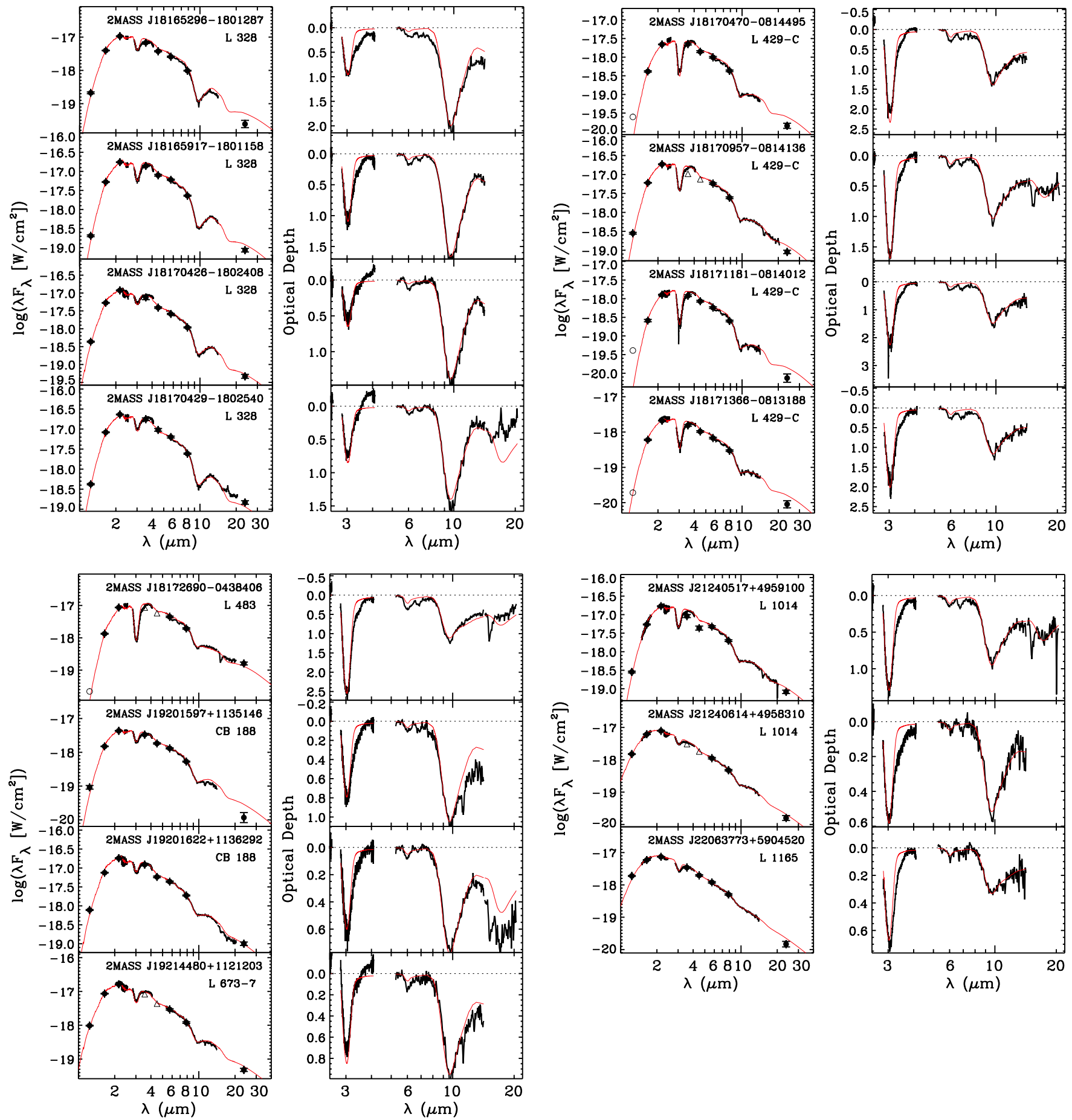

Figure 1. (Continued)

considered (Table 2), as these are bright, and most likely to have made it into the source selection. The blend of $\mathrm{CO}$ overtone lines at $2.25-2.60 \mu \mathrm{m}$ is a sensitive tracer of spectral type. For the later spectral types (>M0 III), the $4.7 \mu \mathrm{m}$ fundamental $\mathrm{CO}$ transitions become prominent as well. Although most of this region was not observed, its edge at 4.0-4.1 $\mu \mathrm{m}$ was; it drops off sharply for spectral types $>$ M0 III and was used as another independent indicator of spectral type. For the earliest spectral types (late G), the $2.16 \mu \mathrm{m} \mathrm{H}-\mathrm{I} \mathrm{Br} \gamma$ line is important. Finally, because the opacity-sampled model spectral lines can only be compared directly to low-resolution observations, the observations and models were smoothed to a resolving power of $R=100$ before fitting them.

2. Continuum extinction. The goal of this work is to measure the strength and shape of dust and ice absorption features, and therefore a continuum-only extinction curve should be applied to the stellar models. Published extinction curves are usually based on broadband photometry (e.g., Indebetouw et al. 2005; Chapman et al. 2009), including continuum and feature extinction and applying large interpolations between, e.g., the IRAC $8 \mu \mathrm{m}$ and MIPS $24 \mu \mathrm{m}$ photometry. Therefore, an independent "high-resolution" extinction curve is derived (Section 4.2), from which a 
Table 2

Model Spectra

\begin{tabular}{lcc}
\hline \hline Spectral Type & $\begin{array}{c}T_{\text {eff }} \\
(\mathrm{K})\end{array}$ & $\log (g)$ \\
\hline G8 III & 5000 & 2.50 \\
K0 III & 4500 & 2.50 \\
K3 III & 4500 & 2.00 \\
K4 III & 4250 & 2.00 \\
K5 III & 4000 & 2.00 \\
K7 III & 4000 & 1.50 \\
M0 III & 3700 & 1.00 \\
M1 III & 3500 & 1.00 \\
M3 III & 3200 & 1.00 \\
M6 III & 3200 & 0.50 \\
M7 III & 3000 & 0.50 \\
M8 III & 2800 & 0.50 \\
M9 III & 2500 & 0.50 \\
\hline
\end{tabular}

Notes. Spectral types were assigned using polynomial fits to empirical $T_{\text {eff }}$ and $\log (g)$ data following the method of Kane \& Sahu (2003).

continuum-only extinction curve was extracted (dashed red line in Figure 2(b)). The latter was applied to the stellar models with $A_{\mathrm{K}}$ as a free parameter.

3. $\mathrm{H}_{2} \mathrm{O}$ ice column density. Broad $\mathrm{H}_{2} \mathrm{O}$ ice features cover much of the observed background star spectra (Figure 2). For sources with observed $L$-band spectra, the strong, relatively sharp $3.0 \mu \mathrm{m}$ band $(\mathrm{O}-\mathrm{H}$ stretch mode) constrains the $\mathrm{H}_{2} \mathrm{O}$ ice column density well. This significantly improves the reliability of the overall fits, because in the 5-8 and 11-20 $\mu \mathrm{m}$ ranges, $\mathrm{H}_{2} \mathrm{O}$ features overlap with other features. Optical constants of amorphous solid $\mathrm{H}_{2} \mathrm{O}$ at $T=10 \mathrm{~K}$ (Hudgins et al. 1993) were used to calculate the absorption spectrum of ice spheres (Bohren \& Huffman 1983). Spheres with radii of $0.4 \mu \mathrm{m}$ fit the typical short-wavelength profile and peak position of the observed $3 \mu \mathrm{m}$ bands best. The peak strengths of the $6.0 \mu \mathrm{m}$ bending and $13 \mu \mathrm{m}$ libration modes relative to the 3.0 stretching mode of this calculated spectrum are $\sim 7 \%$ weaker with respect to the laboratory transmission spectrum (Hudgins et al. 1993). Also, the libration mode peaks at $12.5 \mu \mathrm{m}$, compared to $13.3 \mu \mathrm{m}$ in the laboratory.

4. Silicate feature depth. The contribution of silicate features to the absorption is quantified by the peak optical depth of a synthetic silicate spectrum at $9.7 \mu \mathrm{m}\left(\tau_{9.7}\right)$. The silicate spectrum was calculated for grains small compared to the wavelength (Rayleigh limit) using optical constants (Dorschner et al. 1995) of amorphous pyroxene $\left(\mathrm{Mg}_{0.5} \mathrm{Fe}_{0.5} \mathrm{SiO}_{3}\right)$ and olivine $\left(\mathrm{Mg}_{1} \mathrm{Fe}_{1} \mathrm{SiO}_{4}\right)$. The olivine and pyroxene spectra were added such that the shape of the silicate feature in 2MASS J21240517+4959100 is matched, i.e., $\tau_{9.7}($ pyroxene $) / \tau_{9.7}($ olivine $)=0.62$. Note that this silicate spectrum is broader than the diffuse medium spectrum (Kemper et al. 2004), for which one would need a ratio of 0.20 .

$\chi^{2}$ values were derived for all available observables, including photometric points, ground-based spectra and Spitzer spectra, except for those for which it is a priori known that insufficient information is available: the $3.1-3.7 \mu \mathrm{m}$ spectral region (longwavelength wing $3.0 \mu \mathrm{m}$ band), the 5.3-7.1 $\mu \mathrm{m}$ region $(6.0$ and $6.85 \mu \mathrm{m}$ ice bands), and the IRAC2 photometric point $(4.67 \mu \mathrm{m}$ CO ice band). The MIPS1 photometric point was not fitted

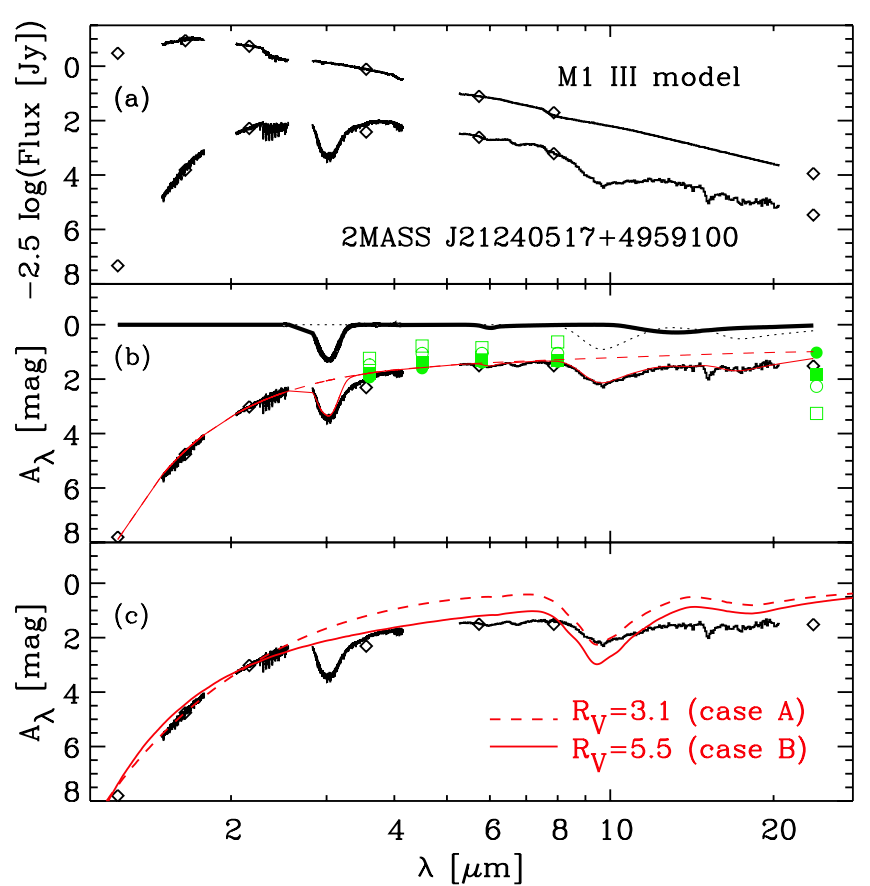

Figure 2. Derivation of an extinction curve for the star 2MASS J21240517+4959100 behind L1014. Panel (a) shows the observed spectrum, broadband photometry (diamonds) and an M1 III model spectrum. After subtracting these spectra on a log scale, the resulting extinction curve is shown in panel (b). The solid red line is the result of adding spectra of solid $\mathrm{H}_{2} \mathrm{O}$ (thick solid black line) and silicate features (dotted black line) to a polynomial fit. The dashed line then represents the feature-free extinction curve used in the background star continuum fits. Green symbols represent the average extinction in broadband filters derived from nearby cloud observations (Chapman et al. 2009) for lines of sight with $A_{\mathrm{K}}<0.5$ (open squares), $0.5<A_{\mathrm{K}}<1.0$ (open circles), $1.0<A_{\mathrm{K}}<2.0$ (filled squares), and $1.0<A_{\mathrm{K}}<2.0$ (filled circles). Panel (c) compares the empirical extinction curve with the calculated curves of $R_{\mathrm{V}}=3.1$ (case A) and 5.5 (case B) from Weingartner \& Draine (2001).

(A color version of this figure is available in the online journal.)

either, to avoid too much weight to the longer wavelengths, which are not important for the ice bands studied here (although, in practice, the final models are in quite good agreement with the MIPS1 photometry; Figure 1). Because there are many more spectroscopic than photometric data points, the latter would have much larger $\chi^{2}$ values. Thus, the spectroscopic $\chi^{2}$ values were divided by the number of data points and then the "reduced $\chi^{2}$ values" of the different observables were averaged to represent the goodness of fit over all observables. In addition, a reduced $\chi^{2}$ value was derived for the most crucial observables for spectral type determination: near-infrared photometry, the shape of nearinfrared spectra and the $4.0-4.1 \mu \mathrm{m}$ spectral region. The reduced $\chi^{2}$ values and parameters of the best fits are reported in Table 3 and the fits are plotted in Figure 1 (red lines in the left panels). Good fits are often obtained. In seven cases, the reduced $\chi^{2}$ values exceed 3 and the main cause for that is indicated in the notes of Table 3 . Typically, the relative scalings of the $K$-band photometry, and the (partial) $K$-band and $L$-band spectra deviate from the model, but the fits are good at longer wavelengths.

In seven other cases, no spectra below $5 \mu \mathrm{m}$, and thus no independent measures of spectral type and $N\left(\mathrm{H}_{2} \mathrm{O}\right)$ were available (Table 1). This most strongly affects the $\mathrm{N}\left(\mathrm{H}_{2} \mathrm{O}\right)$ determination, as the depth of the $13 \mu \mathrm{m}$ libration mode is degenerate with the slope of the applied extinction curve and the assumed silicate band profiles. An uncertainty in the spectral type affects the shape and depth of the 5-8 $\mu \mathrm{m}$ absorption features because of photospheric features that emerge for spectral types later than 
Table 3

Continuum Fit Parameters

\begin{tabular}{|c|c|c|c|c|c|c|}
\hline $\begin{array}{l}\text { Source } \\
\text { 2MASS J }\end{array}$ & Spectral Type ${ }^{a}$ & $\begin{array}{c}A_{\mathrm{K}}^{\mathrm{b}} \\
(\mathrm{mag})\end{array}$ & $\tau_{3.0}{ }^{\mathrm{c}}$ & $\tau_{9.7}{ }^{\mathrm{d}}$ & $\chi_{v}^{2}(\text { total })^{\mathrm{e}}$ & $\chi_{v}^{2}(\mathrm{SpT})^{\mathrm{f}}$ \\
\hline $04215402+1530299$ & K0 (G8-K5) & $3.04(0.09)$ & $1.09(0.05)$ & $0.88(0.04)$ & 1.20 & 1.93 \\
\hline 08052135-3909304 & M0 (K5-M1) & $1.47(0.04)$ & $<0.70^{\mathrm{h}}$ & $0.41(0.02)$ & 0.29 & $\ldots{ }^{\mathrm{i}}$ \\
\hline $08093135-3604035$ & K0 (G8-M1) & $1.89(0.06)$ & $0.55^{\mathrm{h}}(0.25)$ & $0.58(0.02)$ & 0.42 & $\ldots{ }^{i}$ \\
\hline $08093468-3605266$ & M3 (M0-M7) & $4.52(0.14)$ & $2.10^{\mathrm{h}}(0.42)$ & $0.95(0.04)$ & 0.58 & $\ldots i$ \\
\hline $12014301-6508422$ & M3 (M3-M7) & $5.17(0.15)$ & $3.20^{\mathrm{h}}(0.80)$ & $1.35(0.05)$ & 1.94 & $\ldots$ i \\
\hline $12014598-6508586$ & $\mathrm{~K} 3(\mathrm{G} 8-\mathrm{K} 7)^{\mathrm{g}}$ & $3.07(0.09)$ & $1.45^{\mathrm{h}}(0.25)$ & $0.78(0.03)$ & 0.37 & $\ldots i$ \\
\hline $15421547-5248146$ & $\mathrm{M} 0(\mathrm{~K} 7-\mathrm{M} 1)^{\mathrm{g}}$ & $1.94(0.06)$ & $<0.70^{\mathrm{h}}$ & $0.81(0.03)$ & 0.51 & $\ldots \mathrm{i}$ \\
\hline $15421699-5247439$ & K7 (K5-M3) $)^{\mathrm{g}}$ & $2.87(0.09)$ & $0.85^{\mathrm{h}}(0.25)$ & $0.84^{\mathrm{j}}(0.03)$ & 0.26 & $\ldots$ i \\
\hline $17111501-2726180$ & M6 (M3-M6) & $3.91(0.12)$ & $1.39(0.07)$ & $0.76(0.03)$ & 1.21 & 1.92 \\
\hline $17111538-2727144$ & M3 (M1-M6) & $3.53(0.11)$ & $1.20(0.06)$ & $0.87(0.03)$ & 1.96 & 1.69 \\
\hline 17112005-2727131 & M3 (M1-M3) & $6.00(0.18)$ & $2.25(0.11)$ & $1.30(0.05)$ & $4.91^{\mathrm{k}}$ & 5.22 \\
\hline $17155573-2055312$ & M1 (M0-M1) & $2.65(0.08)$ & $0.97(0.05)$ & $0.66(0.03)$ & 1.57 & 1.94 \\
\hline 17160467-2057072 & K7 (K7-M0) & $3.24(0.10)$ & $1.27(0.06)$ & $0.77(0.03)$ & $9.38^{\mathrm{k}}$ & 3.42 \\
\hline 17160860-2058142 & G8 (G8-K4) & $2.45(0.07)$ & $0.91(0.05)$ & $0.77(0.08)$ & 0.72 & 1.28 \\
\hline $18140712-0708413$ & K7 (K5-M0) & $1.89(0.06)$ & $0.83(0.04)$ & $0.60(0.02)$ & 0.64 & 0.72 \\
\hline $18160600-0225539$ & M0 (M0-M1) & $1.25(0.04)$ & $0.63(0.09)$ & $0.50(0.02)$ & 1.77 & 1.76 \\
\hline $18165296-1801287$ & M1 & $3.00(0.18)$ & $0.96(0.10)$ & $2.00(0.12)$ & 1.21 & 1.34 \\
\hline $18165917-1801158$ & M6 (M3-M6) & $3.34(0.10)$ & $1.10(0.05)$ & $1.66(0.07)$ & 0.76 & 1.10 \\
\hline $18170426-1802408$ & M6 (M3-M6) & $2.50(0.08)$ & $0.65(0.03)$ & $1.38(0.06)$ & 1.89 & 4.22 \\
\hline $18170429-1802540$ & M1 (M0-M1) & $2.96(0.09)$ & $0.85(0.09)$ & $1.40(0.06)$ & 2.07 & 1.92 \\
\hline $18170470-0814495$ & M0 (M0-M1) & $4.27(0.13)$ & $2.33(0.12)$ & $1.39(0.06)$ & $12.28^{\mathrm{k}}$ & 2.49 \\
\hline $18170957-0814136$ & M1 (M1-M6) & $3.27(0.10)$ & $1.69(0.08)$ & $1.04(0.04)$ & 2.02 & 2.49 \\
\hline $18171181-0814012$ & K7 (K5-M0) & $4.31(0.13)$ & $2.26(0.11)$ & $1.49(0.06)$ & 2.00 & 1.11 \\
\hline $18171366-0813188$ & K7 (K5-M0) & $3.58(0.11)$ & $2.02(0.10)$ & $1.17(0.05)$ & $16.21^{\mathrm{k}}$ & 1.93 \\
\hline $18172690-0438406$ & M3 (M1-M6) & $4.60(0.14)$ & $2.55(0.13)$ & $1.11(0.04)$ & $4.53^{\mathrm{k}}$ & 0.78 \\
\hline $19201597+1135146$ & M1 (M0-M1) & $3.14(0.09)$ & $0.79(0.04)$ & $1.05(0.04)$ & 1.01 & 0.69 \\
\hline $19201622+1136292$ & M6 (M3-M6) & $2.50(0.13)$ & $0.60(0.03)$ & $0.78(0.03)$ & 0.99 & 1.11 \\
\hline $19214480+1121203$ & M6 (M3-M6) & $2.10(0.06)$ & $0.85(0.04)$ & $0.95(0.04)$ & $7.31^{\mathrm{k}}$ & 1.74 \\
\hline $21240517+4959100$ & M1 (M0-M1) & $3.10(0.09)$ & $1.30(0.06)$ & $0.95(0.04)$ & 1.03 & 0.82 \\
\hline $21240614+4958310$ & K4 (K4-K5) & $1.60(0.05)$ & $0.58(0.03)$ & $0.52(0.02)$ & 1.35 & 1.33 \\
\hline $22063773+5904520$ & G8 & $1.74(0.09)$ & $0.67(0.03)$ & $0.33(0.01)$ & 2.95 & 2.48 \\
\hline $04393886+2611266$ & K7 (K5-M1) & $3.00(0.09)$ & $1.42(0.07)$ & $0.84(0.03)$ & 0.80 & 2.61 \\
\hline $18300061+0115201$ & M3 (M3-M6) & $6.38(0.19)$ & $1.81(0.09)$ & $1.71(0.07)$ & $7.30^{\mathrm{k}}$ & 18.79 \\
\hline
\end{tabular}

Notes.

${ }^{a}$ Best-fitting spectral type that is used throughout this work. The uncertainty range is given in parentheses, unless the spectral type is more accurate than the closest available sub-types. Note that the spectral types are limited to the ones listed in Table 2. All listed spectral types have luminosity class III.

${ }^{\mathrm{b}}$ Extinction in the $K$ band.

${ }^{c}$ Peak absorption optical depth of the $3.0 \mu \mathrm{m} \mathrm{H}_{2} \mathrm{O}$ ice band.

${ }^{\mathrm{d}}$ Peak absorption optical depth of the $9.7 \mu \mathrm{m}$ band of silicates.

${ }^{\mathrm{e}}$ Reduced $\chi^{2}$ values of the model spectrum fitted to all available photometry and spectra.

${ }^{\mathrm{f}}$ Reduced $\chi^{2}$ values of the model spectrum to all available near-infrared photometry and spectra $(J, H, K$, and $L$ bands), excluding the $3.0 \mu \mathrm{m}$ ice band.

${ }^{\mathrm{g}}$ Spectral type determined from wavelengths above $5 \mu \mathrm{m}$ only because no near-infrared spectra available.

h $\tau_{3.0}$ uncertain because no near-infrared spectra available.

${ }^{\mathrm{i}}$ No near-infrared spectra available.

j $9.7 \mu \mathrm{m}$ band narrower than for most other sources in the sample.

${ }^{\mathrm{k}}$ The main reason for large values of $\chi_{v}^{2}$ (total) is different in individual cases: 2MASS J17112005-2727131: only partial $K$-band spectrum available that does not match $K$-band photometry, and only upper limits to 2MASS $J$-and $H$-band photometry available. 2MASS J17160467-2057072: only partial $K$-band spectrum available that does not match $K$-band photometry, possibly due to flux calibration error of the spectrum. 2MASS J18170470-0814495: combined partial $K$ - and $L$-band spectra too high compared to model, uncertainty in IRAC1 photometry underestimated? 2MASS J18171366-0813188: $K$-band spectrum does not match $K$-band photometry, possibly relative $K$ - versus $L$-band calibration error. 2MASS J18172690-0438406: model too shallow between the $H$ and $K$ bands, and too steep above $10 \mu \mathrm{m}$. 2MASS J19214480+1121203: modeled $K$ - and $L$-band spectra systematically too low. 2MASS J18300061+0115201: no IRAC photometry available for scaling the spectra, $H$ - and $K$-band slope steeper than can be modeled.

M1 III. In practice, the $8 \mu \mathrm{m}$ photospheric $\mathrm{SiO}$ bands can be used to constrain the spectral type sufficiently well, however.

\subsection{The "High-resolution" Extinction Curve}

The background star spectra can be used to derive a highresolution extinction curve, in which the ice and dust features can be separated from the continuum extinction and in which large interpolations usually applied between, e.g., the IRAC $8 \mu \mathrm{m}$ and MIPS $24 \mu \mathrm{m}$ photometric data points are avoided. 2MASS J21240517+4959100 behind the core L1014 was chosen for this purpose, because high-quality near-infrared spectra are available, indicating an accurate spectral type of 
Table 4

Polynomial Coefficients of Feature-free Extinction Curve (Equation. (2))

\begin{tabular}{lr}
\hline \hline Coefficient & \multicolumn{1}{c}{ Value } \\
\hline$a_{0}$ & 0.5924 \\
$a_{1}$ & -1.8235 \\
$a_{2}$ & -1.3020 \\
$a_{3}$ & 5.9936 \\
$a_{4}$ & -5.3429 \\
$a_{5}$ & 1.2619 \\
$a_{6}$ & 0.2738 \\
$a_{7}$ & 0.0069 \\
$a_{8}$ & -0.0554 \\
\hline
\end{tabular}

M1 III. The extinction curve was derived as follows:

$$
A_{\lambda}[\mathrm{mag}]=-2.5 \log \left(F_{\lambda}(\mathrm{obs}) / F_{\lambda}(\text { model })\right) .
$$

The absolute scaling of $F_{\lambda}$ (model) is unknown, because there are no measurements of the star's distance. To circumvent this problem, the model was scaled with respect to the observed spectrum such that the derived $A_{\lambda}$ follows known near-infrared extinction curves (i.e., $A_{\lambda} \propto \lambda^{-1.8}$; e.g., Indebetouw et al. 2005). This provides the "high-resolution" extinction curve shown in Figure 2(b). This curve is remarkably flat. At an $A_{\mathrm{K}}$ of $3.02 \mathrm{mag}$, the extinction is still $1.5 \mathrm{mag}$ at $25 \mu \mathrm{m}$. This confirms the results of Chapman et al. (2009), as indicated by the symbols in Figure 2(b), and McClure (2009). Subsequently, continuum and feature extinction were separated by adding a laboratory spectrum of solid $\mathrm{H}_{2} \mathrm{O}$ (at $T=10 \mathrm{~K}$; Hudgins et al. 1993) and a silicate model spectrum (Section 4.1) to a polynomial fit to feature-free regions of the form

$$
\log \left(A_{\lambda} / A_{\mathrm{K}}\right)=a_{0}+a_{1} \log (\lambda)+a_{2}[\log (\lambda)]^{2}+\cdots,
$$

with $\lambda$ in $\mu \mathrm{m}$ and $A_{\lambda}$ in magnitude. The polynomial coefficients are listed in Table 4, and the curve is plotted with a dashed red line in Figure 2(b). This feature-free extinction curve works well for most sources. Examples of exceptions might be 2MASS J19201597+1135146 (model too shallow $>10 \mu \mathrm{m}$ ) and 2MASS J18172690-0438406 (model too steep $>10 \mu \mathrm{m}$ ), but no systematic trend with $A_{\mathrm{K}}$ or core environment could be found. In these cases, the silicate model used in the fits may be responsible for the deviations as well.

\subsection{Absorption Band Strengths and Column Densities}

The continuum fits, as indicated with red lines in Figure 1 (left panels), but excluding modeled $\mathrm{H}_{2} \mathrm{O}$ and silicate bands, were used to put the spectra on an optical depth scale. The right panels of Figure 1 show that the optical depth in the 7.5-8.0 $\mu \mathrm{m}$ region is not always fully explained by the modeled $\mathrm{H}_{2} \mathrm{O}$ and silicate bands. This may be related to inaccuracies in the applied extinction curve and stellar model. Therefore, in the subsequent analysis of the optical depth spectra, local straight line continua between 7.5 and 5.3 or $6.4 \mu \mathrm{m}$ were used to correct for these small offsets (see Figure 3 for example).

Peak and integrated optical depths were determined for the 6.0 and $6.85 \mu \mathrm{m}$ bands, before and after the $\mathrm{O}-\mathrm{H}$ bending mode of $\mathrm{H}_{2} \mathrm{O}$ was subtracted using an amorphous ice spectrum at $T=10 \mathrm{~K}$ (Hudgins et al. 1993; Table 5). The features in the $\mathrm{H}_{2} \mathrm{O}$-subtracted spectra were also decomposed into the five components discussed in Boogert et al. (2008). These components (labeled C1-C5) are useful in characterizing source-to-source variations of the absorption in the 5-7 $\mu \mathrm{m}$ wavelength region

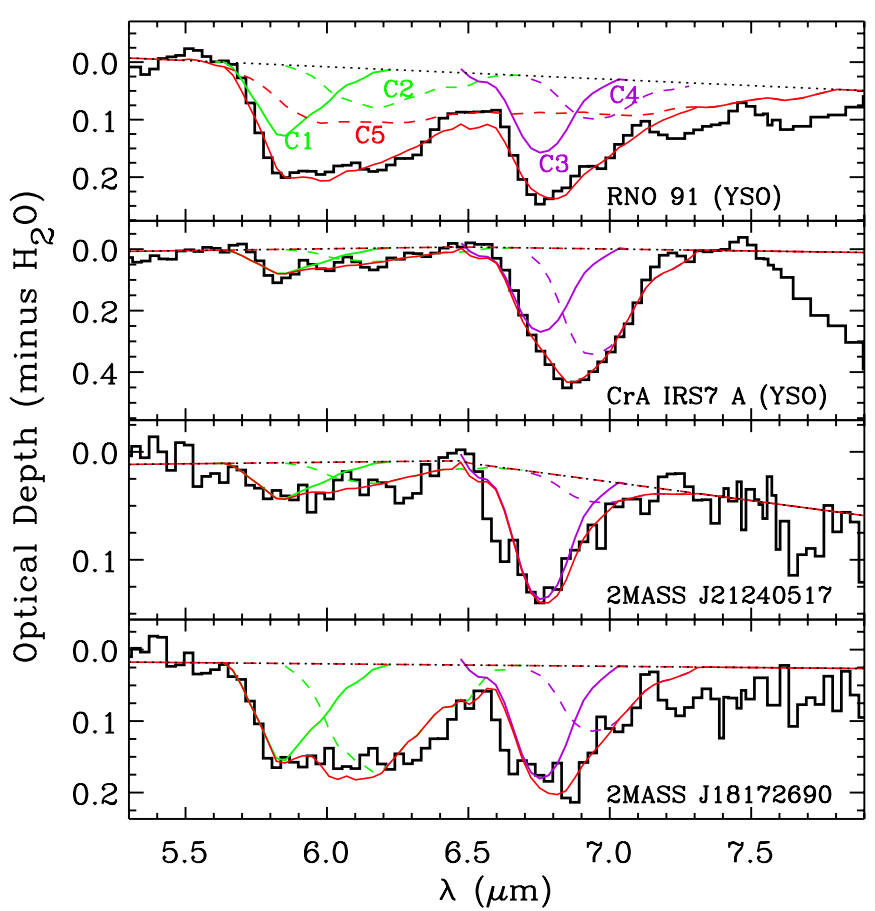

Figure 3. Decomposition of the absorption features in the 5-8 $\mu \mathrm{m}$ spectra of two background stars (bottom panels), compared to two YSOs (Boogert et al. 2008 ) in the top panels. The components are labeled C1 (solid green), C2 (dashed green), C3 (solid purple), C4 (dashed purple), and C5 (dashed red). The sum of the components is indicated with a thick red line. Note that the relative strengths of the components (e.g., C3/C1) varies strongly toward both YSOs and background stars. Component $\mathrm{C} 5$ has not been detected toward any background star. All components are added to a local straight line continuum (dotted black line) to remove small inaccuracies from the global continuum-fitting process.

(A color version of this figure is available in the online journal.)

(Section 4.4.6). They do not represent a unique decomposition, however, and each component may be the result of absorption by more than one molecular band. Examples of this decomposition are shown in Figure 3. For comparison, two YSOs are shown as well (Boogert et al. 2008). Clearly, the relative depths of the components vary considerably between background stars and between YSOs.

Column densities were determined for solid $\mathrm{H}_{2} \mathrm{O}, \mathrm{NH}_{4}^{+}$, and $\mathrm{CO}_{2}$ (Table 6), using the intrinsic integrated band strengths listed in Table 7. The $\mathrm{H}_{2} \mathrm{O}$ column density is unreliable if no $L$-band spectrum is available ( 7 out of 31 sources), and the $13 \mu \mathrm{m}$ libration mode is the main tracer. The depth of the latter is strongly affected by the assumed shape of the extinction curve and by overlapping stretching and bending modes of silicates. The $\mathrm{NH}_{4}^{+}$column density is derived, assuming that the entire $6.85 \mu \mathrm{m}$ band (i.e., both components "C3" and "C4" listed in Table 5) can be attributed to it, after correction for any overlapping bands of the $\mathrm{C}-\mathrm{H}$ deformation mode of solid $\mathrm{CH}_{3} \mathrm{OH}$ (see below). This identification has not been fully validated, however, because no other $\mathrm{NH}_{4}^{+}$bands have been detected (Schutte \& Khanna 2003; Boogert et al. 2008). The identification of the $15.2 \mu \mathrm{m}$ band is not disputed, and the solid $\mathrm{CO}_{2}$ column density is listed for the eight sources for which this wavelength range was observed. All $\mathrm{CO}_{2}$ observations were done at a low resolving power of $\sim 90$, which is insufficient to assess the composition or thermal processing history of the ices (Pontoppidan et al. 2008).

The background star spectra were also searched for $\mathrm{CH}_{3} \mathrm{OH}$, $\mathrm{HCOOH}, \mathrm{NH}_{3}$, and $\mathrm{CH}_{4}$ species that are often detected toward YSOs. For solid $\mathrm{CH}_{3} \mathrm{OH}$, the $3.53 \mu \mathrm{m} \mathrm{C}-\mathrm{H}$ stretching and the 
Table 5

Optical Depths 5-8 $\mu \mathrm{m}$ Features

\begin{tabular}{|c|c|c|c|c|c|c|c|c|c|c|c|}
\hline $\begin{array}{l}\text { Source } \\
\text { 2MASS J }\end{array}$ & $\begin{array}{c}\tau_{\text {int }}(5.2-6.4 \mu \mathrm{m})^{\mathrm{a}} \\
\left(\mathrm{cm}^{-1}\right)\end{array}$ & $\begin{array}{c}\tau_{\text {int }}(5.2-6.4 \mu \mathrm{m})^{\mathrm{b}} \\
\text { Minus } \mathrm{H}_{2} \mathrm{O} \\
\left(\mathrm{cm}^{-1}\right)\end{array}$ & $\tau_{\mathrm{int}}(6.4-7.2 \mu \mathrm{m})^{\mathrm{c}}$ & $\begin{array}{c}\tau_{\text {int }}(6.4-7.2 \mu \mathrm{m})^{\mathrm{d}} \\
\text { Minus H} \mathrm{H}_{2} \mathrm{O} \\
\left(\mathrm{cm}^{-1}\right)\end{array}$ & $\tau_{6.0} \mathrm{e}$ & $\tau_{6.85^{f}}$ & $\tau_{\mathrm{C} 1}^{\mathrm{g}}$ & $\tau_{\mathrm{C} 2}{ }^{\mathrm{h}}$ & $\tau_{\mathrm{C} 3}{ }^{\mathrm{i}}$ & $\tau_{C 4}{ }^{j}$ & $\tau_{\mathrm{C} 5}{ }^{\mathrm{k}}$ \\
\hline $04215402+1530299$ & $26.27(1.96)$ & $14.06(1.96)$ & $17.48(2.69)$ & $12.44(2.69)$ & $0.19(0.04)$ & $0.15(0.07)$ & $0.08(0.01)$ & $0.09(0.01)$ & $0.10(0.03)$ & $0.08(0.03)$ & $0.00(0.05)$ \\
\hline $08052135-3909304$ & $6.57(0.94)$ & $-1.26(0.94)$ & $7.54(1.10)$ & $4.31(1.10)$ & $0.05(0.02)$ & $0.06(0.03)$ & $0.00(0.01)$ & $0.00(0.01)$ & $0.03(0.01)$ & $0.03(0.01)$ & $0.00(0.02)$ \\
\hline $08093135-3604035$ & $10.44(1.02)$ & $4.30(1.02)$ & $9.36(2.68)$ & $6.83(2.68)$ & $0.10(0.02)$ & $0.09(0.03)$ & $0.01(0.01)$ & $0.04(0.01)$ & $0.05(0.01)$ & $0.04(0.01)$ & $0.00(0.03)$ \\
\hline $08093468-3605266$ & $50.63(1.71)$ & $27.16(1.71)$ & $22.61(2.24)$ & $12.94(2.24)$ & $0.33(0.04)$ & $0.17(0.05)$ & $0.14(0.01)$ & $0.18(0.01)$ & $0.09(0.02)$ & $0.08(0.02)$ & $0.00(0.04)$ \\
\hline $12014301-6508422$ & $49.01(1.05)$ & $13.22(1.05)$ & $19.23(1.32)$ & $4.49(1.32)$ & $0.33(0.02)$ & $0.19(0.04)$ & $0.02(0.01)$ & $0.12(0.01)$ & $0.10(0.02)$ & $0.00(0.01)$ & $0.00(0.04)$ \\
\hline $12014598-6508586$ & $26.41(1.09)$ & $10.20(1.09)$ & $20.76(1.38)$ & $14.08(1.38)$ & $0.18(0.02)$ & $0.16(0.04)$ & $0.07(0.01)$ & $0.06(0.01)$ & $0.13(0.02)$ & $0.07(0.01)$ & $0.00(0.03)$ \\
\hline $15421547-5248146$ & $8.36(2.18)$ & $0.54(2.18)$ & $6.47(2.54)$ & $3.25(2.54)$ & $0.06(0.04)$ & $0.07(0.06)$ & $0.01(0.01)$ & $0.00(0.02)$ & $0.03(0.02)$ & $0.00(0.03)$ & $0.00(0.05)$ \\
\hline $15421699-5247439$ & $15.41(1.26)$ & $5.90(1.26)$ & $12.40(1.65)$ & $8.49(1.65)$ & $0.11(0.03)$ & $0.10(0.04)$ & $0.07(0.01)$ & $0.01(0.01)$ & $0.06(0.02)$ & $0.06(0.02)$ & $0.00(0.03)$ \\
\hline $17111501-2726180$ & $18.76(0.64)$ & $3.17(0.64)$ & $13.70(0.80)$ & $7.28(0.80)$ & $0.14(0.01)$ & $0.12(0.02)$ & $0.03(0.01)$ & $0.02(0.01)$ & $0.09(0.01)$ & $0.03(0.01)$ & $0.00(0.02)$ \\
\hline $17111538-2727144$ & $24.14(2.75)$ & $10.77(2.75)$ & $8.11(3.48)$ & $2.60(3.48)$ & $0.20(0.06)$ & $0.08(0.09)$ & $0.06(0.02)$ & $0.05(0.02)$ & $0.01(0.04)$ & $0.03(0.04)$ & $0.00(0.07)$ \\
\hline $17112005-2727131$ & $46.71(1.95)$ & $21.56(1.95)$ & $20.81(2.21)$ & $10.44(2.21)$ & $0.30(0.04)$ & $0.20(0.06)$ & $0.11(0.01)$ & $0.13(0.01)$ & $0.13(0.02)$ & $0.04(0.02)$ & $0.00(0.04)$ \\
\hline $17155573-2055312$ & $14.83(1.60)$ & $4.00(1.60)$ & $8.26(1.97)$ & $3.80(1.97)$ & $0.11(0.03)$ & $0.08(0.05)$ & $0.04(0.01)$ & $0.02(0.01)$ & $0.06(0.02)$ & $0.00(0.02)$ & $0.00(0.04)$ \\
\hline $17160467-2057072$ & $14.30(2.48)$ & $0.15(2.48)$ & $19.51(3.38)$ & $13.67(3.38)$ & $0.10(0.05)$ & $0.18(0.08)$ & $0.00(0.02)$ & $0.01(0.02)$ & $0.11(0.03)$ & $0.10(0.03)$ & $0.00(0.07)$ \\
\hline $17160860-2058142$ & $11.18(1.31)$ & $1.00(1.31)$ & $7.75(1.78)$ & $3.55(1.78)$ & $0.08(0.03)$ & $0.08(0.05)$ & $0.00(0.01)$ & $0.01(0.01)$ & $0.05(0.02)$ & $0.01(0.02)$ & $0.00(0.03)$ \\
\hline $18140712-0708413$ & $13.30(1.77)$ & $3.98(1.77)$ & $10.78(1.61)$ & $6.94(1.61)$ & $0.09(0.03)$ & $0.07(0.04)$ & $0.02(0.01)$ & $0.04(0.01)$ & $0.04(0.02)$ & $0.04(0.02)$ & $0.00(0.04)$ \\
\hline $18160600-0225539$ & $14.64(1.66)$ & $7.60(1.66)$ & $11.23(2.21)$ & $8.33(2.21)$ & $0.10(0.03)$ & $0.09(0.06)$ & $0.05(0.01)$ & $0.04(0.01)$ & $0.04(0.02)$ & $0.06(0.02)$ & $0.00(0.04)$ \\
\hline $18165296-1801287$ & $19.54(0.92)$ & $8.81(0.92)$ & $8.00(1.24)$ & $3.58(1.24)$ & $0.13(0.02)$ & $0.09(0.03)$ & $0.04(0.01)$ & $0.06(0.01)$ & $0.06(0.01)$ & $0.01(0.01)$ & $0.00(0.03)$ \\
\hline $18165917-1801158$ & $24.64(1.05)$ & $12.37(1.05)$ & $12.63(1.22)$ & $7.58(1.22)$ & $0.17(0.02)$ & $0.11(0.03)$ & $0.05(0.01)$ & $0.10(0.01)$ & $0.08(0.01)$ & $0.04(0.01)$ & $0.00(0.02)$ \\
\hline $18170426-1802408$ & $23.25(1.18)$ & $15.98(1.18)$ & $14.30(1.46)$ & $11.31(1.46)$ & $0.17(0.02)$ & $0.10(0.04)$ & $0.07(0.01)$ & $0.12(0.01)$ & $0.08(0.01)$ & $0.06(0.02)$ & $0.00(0.03)$ \\
\hline $18170429-1802540$ & $17.86(2.29)$ & $8.34(2.29)$ & $9.63(2.10)$ & $5.72(2.10)$ & $0.13(0.05)$ & $0.09(0.05)$ & $0.03(0.01)$ & $0.07(0.02)$ & $0.06(0.02)$ & $0.04(0.02)$ & $0.00(0.05)$ \\
\hline $18170470-0814495$ & $40.68(1.48)$ & $14.60(1.48)$ & $23.81(1.85)$ & $13.06(1.85)$ & $0.28(0.03)$ & $0.21(0.05)$ & $0.11(0.01)$ & $0.07(0.01)$ & $0.15(0.02)$ & $0.06(0.02)$ & $0.00(0.04)$ \\
\hline $18170957-0814136$ & $27.44(1.10)$ & $8.56(1.10)$ & $18.09(1.22)$ & $10.31(1.22)$ & $0.19(0.02)$ & $0.16(0.03)$ & $0.07(0.01)$ & $0.03(0.01)$ & $0.11(0.01)$ & $0.06(0.01)$ & $0.00(0.02)$ \\
\hline $18171181-0814012$ & $41.27(1.68)$ & $15.97(1.68)$ & $26.33(2.23)$ & $15.90(2.23)$ & $0.28(0.04)$ & $0.23(0.06)$ & $0.12(0.01)$ & $0.07(0.01)$ & $0.15(0.02)$ & $0.11(0.02)$ & $0.00(0.04)$ \\
\hline $18171366-0813188$ & $34.05(1.63)$ & $11.50(1.63)$ & $25.80(2.12)$ & $16.50(2.12)$ & $0.24(0.04)$ & $0.21(0.06)$ & $0.08(0.01)$ & $0.06(0.01)$ & $0.15(0.02)$ & $0.10(0.02)$ & $0.00(0.04)$ \\
\hline $18172690-0438406$ & $52.65(1.15)$ & $24.10(1.15)$ & $28.26(1.34)$ & $16.49(1.34)$ & $0.34(0.03)$ & $0.23(0.04)$ & $0.14(0.01)$ & $0.15(0.01)$ & $0.16(0.02)$ & $0.09(0.01)$ & $0.00(0.03)$ \\
\hline $19201597+1135146$ & $14.91(1.20)$ & $6.04(1.20)$ & $10.25(1.72)$ & $6.59(1.72)$ & $0.10(0.03)$ & $0.09(0.04)$ & $0.03(0.01)$ & $0.04(0.01)$ & $0.08(0.02)$ & $0.04(0.02)$ & $0.00(0.04)$ \\
\hline $19201622+1136292$ & $11.74(0.76)$ & $5.00(0.76)$ & $4.40(0.77)$ & $1.62(0.77)$ & $0.09(0.01)$ & $0.05(0.02)$ & $0.04(0.01)$ & $0.03(0.01)$ & $0.03(0.01)$ & $0.00(0.01)$ & $0.00(0.02)$ \\
\hline $19214480+1121203$ & $19.51(1.72)$ & $10.01(1.72)$ & $10.35(2.22)$ & $6.43(2.22)$ & $0.15(0.04)$ & $0.09(0.06)$ & $0.04(0.01)$ & $0.08(0.01)$ & $0.02(0.02)$ & $0.06(0.02)$ & $0.00(0.04)$ \\
\hline $21240517+4959100$ & $19.44(1.23)$ & $4.93(1.23)$ & $13.95(1.31)$ & $7.97(1.31)$ & $0.13(0.02)$ & $0.14(0.03)$ & $0.03(0.01)$ & $0.02(0.01)$ & $0.12(0.01)$ & $0.02(0.01)$ & $0.00(0.02)$ \\
\hline $21240614+4958310$ & $8.54(1.26)$ & $2.01(1.26)$ & $8.12(1.54)$ & $5.44(1.54)$ & $0.08(0.03)$ & $0.08(0.04)$ & $0.00(0.01)$ & $0.02(0.01)$ & $0.03(0.02)$ & $0.06(0.02)$ & $0.00(0.03)$ \\
\hline $22063773+5904520$ & $10.22(1.31)$ & $2.70(1.31)$ & $9.70(1.73)$ & $6.61(1.73)$ & $0.09(0.03)$ & $0.08(0.05)$ & $0.00(0.01)$ & $0.03(0.01)$ & $0.05(0.02)$ & $0.05(0.02)$ & $0.00(0.03)$ \\
\hline $04393886+2611266$ & $20.66(0.22)$ & $4.78(0.22)$ & $13.48(0.29)$ & $6.93(0.29)$ & $0.15(0.01)$ & $0.12(0.01)$ & $0.03(0.01)$ & $0.02(0.01)$ & $0.08(0.01)$ & $0.04(0.01)$ & $0.00(0.01)$ \\
\hline $18300061+0115201$ & $33.96(1.25)$ & $13.77(1.25)$ & $20.82(0.89)$ & $12.51(0.89)$ & $0.26(0.03)$ & $0.20(0.02)$ & $0.10(0.01)$ & $0.08(0.01)$ & $0.14(0.01)$ & $0.08(0.01)$ & $0.00(0.03)$ \\
\hline
\end{tabular}

Notes. Uncertainties in parentheses based on statistical errors in the spectra only, unless noted otherwise below.

${ }^{a}$ Integrated optical depth between 5.2 and $6.4 \mu \mathrm{m}$ in wavenumber units.

${ }^{\mathrm{b}}$ Integrated optical depth between 5.2 and $6.4 \mu \mathrm{m}$ in wavenumber units, after subtraction of a laboratory spectrum of pure $\mathrm{H}_{2} \mathrm{O}$ ice.

${ }^{\mathrm{c}}$ Integrated optical depth between 6.4 and $7.2 \mu \mathrm{m}$ in wavenumber units.

${ }^{\mathrm{d}}$ Integrated optical depth between 6.4 and $7.2 \mu \mathrm{m}$ in wavenumber units, after subtraction of a laboratory spectrum of pure $\mathrm{H}_{2} \mathrm{O}$ ice.

e Peak optical depth at $6.0 \mu \mathrm{m}$.

${ }^{\mathrm{f}}$ Peak optical depth at $6.85 \mu \mathrm{m}$.

g Peak optical depth component C1 (Boogert et al. 2008).

${ }^{\text {h }}$ Peak optical depth component $\mathrm{C} 2$

${ }^{\text {i }}$ Peak optical depth component C3.

j Peak optical depth component $\mathrm{C} 4$

${ }^{\mathrm{k}}$ Peak optical depth component $\mathrm{C} 5$. Uncertainty includes uncertainty in $N\left(\mathrm{H}_{2} \mathrm{O}\right)$ as this affects the baseline level. 


\begin{tabular}{|c|c|c|c|c|c|c|c|c|c|c|c|c|c|}
\hline \multirow{2}{*}{$\begin{array}{l}\text { Source } \\
\text { 2MASS J }\end{array}$} & \multirow{2}{*}{$\begin{array}{c}N\left(\mathrm{H}_{2} \mathrm{O}\right)^{\mathrm{a}} \\
10^{18} \mathrm{~cm}^{-2}\end{array}$} & \multicolumn{2}{|c|}{$N\left(\mathrm{NH}_{4}^{+}\right)^{\mathrm{b}}$} & \multicolumn{2}{|c|}{$N\left(\mathrm{CO}_{2}\right)$} & \multicolumn{2}{|c|}{$N\left(\mathrm{CH}_{3} \mathrm{OH}\right)^{\mathrm{c}}$} & \multicolumn{2}{|c|}{$N(\mathrm{HCOOH})$} & \multicolumn{2}{|c|}{$N\left(\mathrm{CH}_{4}\right)$} & \multicolumn{2}{|c|}{$N\left(\mathrm{NH}_{3}\right)$} \\
\hline & & $10^{17} \mathrm{~cm}^{-2}$ & $\% \mathrm{H}_{2} \mathrm{O}$ & $10^{17} \mathrm{~cm}^{-2}$ & $\% \mathrm{H}_{2} \mathrm{O}$ & $10^{17} \mathrm{~cm}^{-2}$ & $\% \mathrm{H}_{2} \mathrm{O}$ & $10^{17} \mathrm{~cm}^{-2}$ & $\% \mathrm{H}_{2} \mathrm{O}$ & $10^{17} \mathrm{~cm}^{-2}$ & $\% \mathrm{H}_{2} \mathrm{O}$ & $10^{17} \mathrm{~cm}^{-2}$ & $\% \mathrm{H}_{2} \mathrm{O}$ \\
\hline $04215402+1530299$ & $1.84(0.20)$ & $2.73(0.61)$ & $14.80(3.71)$ & $\ldots$ & & $<1.55$ & $<9.46$ & $<0.98$ & $<6.03$ & $<1.54$ & $<9.45$ & $<5.46$ & $<33.38$ \\
\hline $08052135-3909304$ & $<1.18^{\mathrm{d}}$ & $0.98(0.24)$ & $>8.29$ & 3.79 & $>32.11$ & $<0.50$ & $\ldots$ & $<0.23$ & $\ldots$ & $<1.02$ & $\ldots$ & $<2.00$ & $\ldots$ \\
\hline $08093135-3604035$ & $0.92(0.42)^{\mathrm{d}}$ & $1.55(0.60)$ & $16.71(10.1)$ & $<11.31$ & $<226.0$ & $<2.00$ & $<39.97$ & $<0.23$ & $<4.61$ & $<2.15$ & $<43.03$ & $<3.00$ & $<59.95$ \\
\hline $08093468-3605266$ & $3.54(0.79)^{\mathrm{d}}$ & $2.94(0.50)$ & $8.29(2.34)$ & $\ldots$ & $\ldots$ & $<10.00$ & $<36.34$ & $<1.63$ & $<5.94$ & $<1.86$ & $<6.79$ & $<4.13$ & $<15.04$ \\
\hline $12014301-6508422$ & $5.40(1.45)^{\mathrm{d}}$ & $1.02(0.30)$ & $1.89(0.75)$ & $\ldots$ & $\ldots$ & $<8.52$ & $<21.59$ & $<0.29$ & $<0.75$ & $<1.15$ & $<2.92$ & $<9.00$ & $<22.80$ \\
\hline $12014598-6508586$ & $2.44(0.48)^{\mathrm{d}}$ & $3.20(0.31)$ & $13.07(2.87)$ & $8.34(1.91)$ & $34.11(10.3)$ & $<2.00$ & $<10.18$ & $<0.81$ & $<4.17$ & $<1.01$ & $<5.17$ & $<4.00$ & $<20.36$ \\
\hline $15421547-5248146$ & $<1.18^{\mathrm{d}}$ & $0.73(0.57)$ & $>6.25$ & $\ldots$ & $\ldots$ & $<5.34$ & $\ldots$ & $<0.44$ & & $<2.03$ & 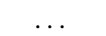 & $<3.64$ & $\ldots$ \\
\hline $15421699-5247439$ & $1.43(0.44)^{\mathrm{d}}$ & $1.92(0.37)$ & $13.45(4.88)$ & $\ldots$ & $\ldots$ & $<2.74$ & $<27.62$ & $<0.81$ & $<8.22$ & $<2.08$ & $<20.99$ & $<4.06$ & $<40.83$ \\
\hline $17111501-2726180$ & $2.35(0.26)$ & $1.52(0.18)$ & $6.49(1.06)$ & $\ldots$ & $\ldots$ & $<1.60$ & $<7.65$ & $<0.35$ & $<1.70$ & $<0.57$ & $<2.75$ & $<1.32$ & $<6.32$ \\
\hline $17111538-2727144$ & $2.01(0.22)$ & $<2.37$ & $<13.24$ & $\ldots$ & $\ldots$ & $<2.00$ & $<11.15$ & $<0.76$ & $<4.27$ & $<2.58$ & $<14.41$ & $<3.00$ & $<16.73$ \\
\hline $17112005-2727131$ & $3.79(0.42)$ & $2.17(0.50)$ & $5.71(1.46)$ & $\ldots$ & $\ldots$ & $<2.00$ & $<5.92$ & $<1.34$ & $<3.99$ & $<1.39$ & $<4.14$ & $<9.00$ & $<26.68$ \\
\hline $17155573-2055312$ & $1.63(0.18)$ & $0.77(0.44)$ & $4.74(2.79)$ & $\ldots$ & $\ldots$ & $<0.86$ & $<5.94$ & $<0.42$ & $<2.90$ & $<1.41$ & $<9.71$ & $<4.00$ & $<27.54$ \\
\hline $17160467-2057072$ & $2.13(0.23)$ & $2.99(0.76)$ & $13.99(3.91)$ & $\ldots$ & $\ldots$ & $<1.39$ & $<7.32$ & $<0.58$ & $<3.09$ & $<1.81$ & $<9.54$ & $<4.00$ & $<21.06$ \\
\hline 17160860-2058142 & $1.53(0.17)$ & $0.72(0.40)$ & $4.72(2.69)$ & $\ldots$ & $\ldots$ & $<0.43$ & $<3.17$ & $<0.31$ & $<2.32$ & $<1.10$ & $<8.10$ & $<4.00$ & $<29.30$ \\
\hline $18140712-0708413$ & $1.40(0.15)$ & $1.40(0.36)$ & $9.99(2.83)$ & $\ldots$ & $\ldots$ & $0.70(0.20)$ & $4.97(1.52)$ & $<0.30$ & $<2.46$ & $<1.05$ & $<8.45$ & $<3.50$ & $<27.99$ \\
\hline $18160600-0225539$ & $1.06(0.19)$ & $1.83(0.50)$ & $17.29(5.65)$ & $\ldots$ & $\ldots$ & $<0.76$ & $<8.73$ & $<0.56$ & $<6.47$ & $<1.64$ & $<18.86$ & $<3.50$ & $<40.18$ \\
\hline $18165296-1801287$ & $1.62(0.22)$ & $0.72(0.28)$ & $4.48(1.84)$ & $\ldots$ & $\ldots$ & $<1.36$ & $<9.82$ & $<0.48$ & $<3.49$ & $<1.20$ & $<8.62$ & $<2.48$ & $<17.87$ \\
\hline $18165917-1801158$ & $1.85(0.20)$ & $1.62(0.27)$ & $8.76(1.78)$ & $\ldots$ & $\ldots$ & $<0.85$ & $<5.21$ & $<0.56$ & $<3.43$ & $<0.71$ & $<4.36$ & $<3.32$ & $<20.22$ \\
\hline $18170426-1802408$ & $1.09(0.12)$ & $2.51(0.33)$ & $22.90(3.96)$ & $\ldots$ & $\ldots$ & $<0.83$ & $<8.60$ & $<0.83$ & $<8.61$ & $<0.96$ & $<9.85$ & $<3.72$ & $<38.23$ \\
\hline $18170429-1802540$ & $1.43(0.20)$ & $1.22(0.47)$ & $8.52(3.53)$ & $3.37(1.95)$ & $23.55(14.0)$ & $<0.77$ & $<6.29$ & $<0.37$ & $<3.05$ & $<0.90$ & $<7.32$ & $<3.67$ & $<29.82$ \\
\hline $18170470-0814495$ & $3.93(0.44)$ & $2.41(0.42)$ & $6.13(1.26)$ & $\ldots$ & & $2.49(0.60)$ & $6.34(1.69)$ & $<1.28$ & $<3.66$ & $<1.80$ & $<5.14$ & $<7.00$ & $<20.00$ \\
\hline $18170957-0814136$ & $2.85(0.31)$ & $1.82(0.27)$ & $6.39(1.20)$ & $12.29(1.29)$ & $43.12(6.63)$ & $2.69(0.58)$ & $9.46(2.30)$ & $<0.89$ & $<3.53$ & $<0.97$ & $<3.84$ & $<3.16$ & $<12.48$ \\
\hline $18171181-0814012$ & $3.81(0.42)$ & $2.80(0.50)$ & $7.35(1.55)$ & $\ldots$ & $\ldots$ & $4.42(0.86)$ & $11.58(2.60)$ & $<1.48$ & $<4.38$ & $<1.42$ & $<4.19$ & $<6.71$ & $<19.80$ \\
\hline $18171366-0813188$ & $3.40(0.38)$ & $3.09(0.48)$ & $9.08(1.74)$ & $\ldots$ & & $3.48(0.86)$ & $10.24(2.78)$ & $<0.99$ & $<3.29$ & $<1.24$ & $<4.12$ & $<4.83$ & $<15.97$ \\
\hline $18172690-0438406$ & $4.31(0.48)$ & $3.02(0.30)$ & $7.01(1.05)$ & $18.86(1.72)$ & $43.75(6.31)$ & $3.63(0.65)$ & $8.44(1.79)$ & $<1.63$ & $<4.28$ & $<0.95$ & $<2.48$ & $<7.00$ & $<18.28$ \\
\hline $19201597+1135146$ & $1.34(0.14)$ & $1.42(0.39)$ & $10.64(3.14)$ & $\ldots$ & & $<0.70$ & $<5.95$ & $<0.37$ & $<3.12$ & $<1.15$ & $<9.69$ & $<6.58$ & $<55.33$ \\
\hline $19201622+1136292$ & $1.01(0.11)$ & $0.31(0.17)$ & $3.09(1.74)$ & $3.86(1.07)$ & $38.08(11.3)$ & $<0.70$ & $<7.77$ & $<0.50$ & $<5.58$ & $<0.58$ & $<6.49$ & $<2.00$ & $<22.15$ \\
\hline $19214480+1121203$ & $1.43(0.16)$ & $1.38(0.50)$ & $9.65(3.68)$ & $\ldots$ & $\ldots$ & $<1.61$ & $<12.64$ & $<0.49$ & $<3.88$ & $<1.63$ & $<12.85$ & $<2.57$ & $<20.22$ \\
\hline $21240517+4959100$ & $2.19(0.24)$ & $1.48(0.29)$ & $6.76(1.55)$ & $8.24(2.69)$ & $37.60(13.0)$ & $1.54(0.38)$ & $7.04(1.90)$ & $<0.40$ & $<2.07$ & $<1.83$ & $<9.43$ & $<4.20$ & $<21.57$ \\
\hline $21240614+4958310$ & $0.98(0.11)$ & $1.18(0.34)$ & $12.01(3.78)$ & ... & $\ldots$ & $<0.59$ & $<6.83$ & $<0.27$ & $<3.13$ & $<1.14$ & $<13.04$ & $<2.50$ & $<28.57$ \\
\hline $22063773+5904520$ & $1.13(0.12)$ & $1.44(0.39)$ & $12.70(3.74)$ & $\ldots$ & $\ldots$ & $<0.40$ & $<3.98$ & $<0.31$ & $<3.10$ & $<1.11$ & $<11.02$ & $<2.30$ & $<22.82$ \\
\hline $04393886+2611266$ & $2.39(0.26)$ & $1.44(0.06)$ & $6.03(0.72)$ & $7.11(0.05)$ & $29.66(3.32)$ & $<0.25$ & $<1.17$ & $<0.38$ & $<1.81$ & $<0.82$ & $<3.84$ & $<1.45$ & $<6.85$ \\
\hline $18300061+0115201$ & $3.04(0.34)$ & $2.68(0.20)$ & $8.79(1.18)$ & $11.93(0.21)$ & $39.14(4.43)$ & $<2.10$ & $<7.75$ & $<1.18$ & $<4.36$ & $<0.58$ & $<2.14$ & $<6.39$ & $<23.61$ \\
\hline
\end{tabular}

Notes. Column densities were determined using the intrinsic integrated band strengths listed in Table 7. Uncertainties $(1 \sigma)$ are indicated in brackets and upper limits are of $3 \sigma$ significance.

${ }^{a}$ An uncertainty of $10 \%$ in the intrinsic integrated band strength is taken into account in the listed column density uncertainties.

b Assuming both $\mathrm{C} 3$ and $\mathrm{C} 4$ components (Boogert et al. 2008) are due to $\mathrm{NH}_{4}^{+}$(Section 4.3). The contribution by the $\mathrm{CH}_{3} \mathrm{OH} \mathrm{C}-\mathrm{H}$ bending mode has been subtracted for sources with $\mathrm{CH} \mathrm{H}_{3} \mathrm{OH}$ detections.

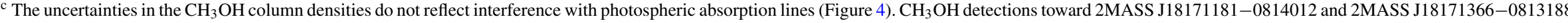
are most reliable.

${ }^{d}$ No $L$-band spectrum available for $\mathrm{H}_{2} \mathrm{O}$ ice column density determination. The $13 \mu \mathrm{m} \mathrm{H} \mathrm{H}_{2} \mathrm{O}$ libration mode was used instead. These values are uncertain, and the listed uncertainties are estimates. 


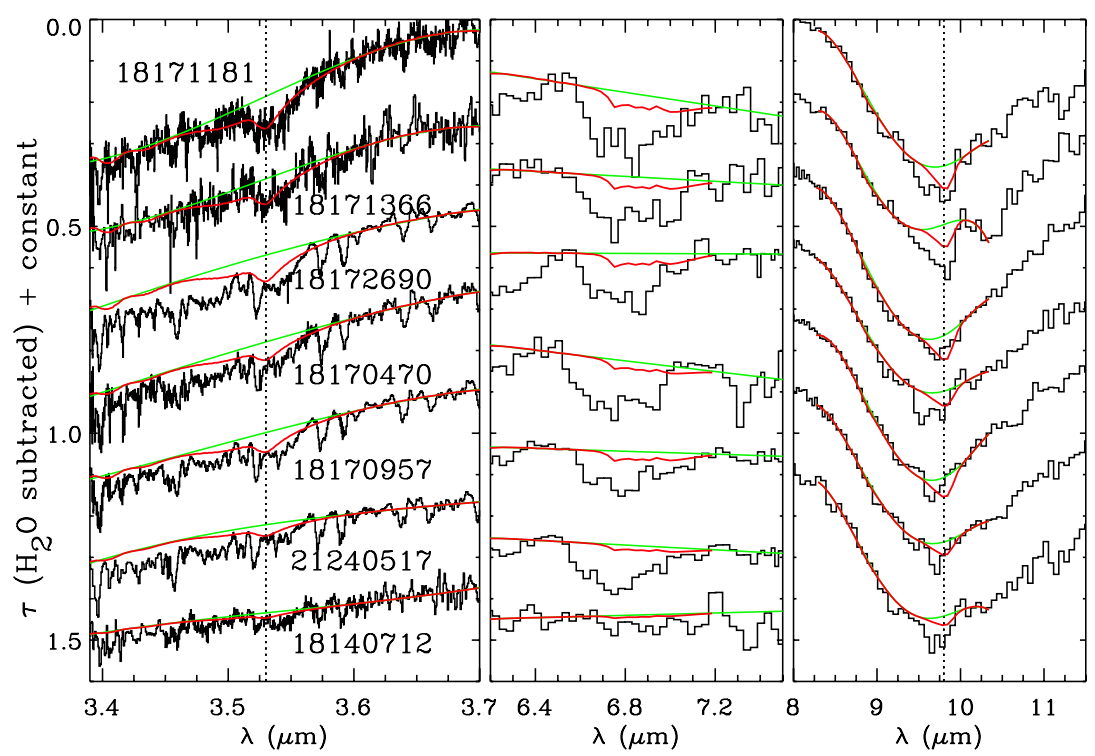

Figure 4. Left panel: sources with detections (top two sources) or tentative detections of the $\mathrm{C}-\mathrm{H}$ stretching mode of solid $\mathrm{CH}_{3} \mathrm{OH}$ at $3.53 \mu \mathrm{m}$. The green line is the assumed local continuum and the red line the laboratory spectrum $\mathrm{H}_{2} \mathrm{O}: \mathrm{CH}_{3} \mathrm{OH}: \mathrm{CO}: \mathrm{NH}_{3}=100: 10: 1: 1(T=10 \mathrm{~K}$; Hudgins et al. 1993) corresponding to the column densities listed in Table 6. Middle panel: the 6.3-8.0 $\mu \mathrm{m}$ region of the same sources as in the left panel showing the $\mathrm{C}-\mathrm{H}$ bending mode of $\mathrm{CH}_{3} \mathrm{OH}$ (red) with the assumed local continuum (green) at the same column densities as in the left panel. The observed absorption is much deeper due to an overlapping band of (possibly) $\mathrm{NH}_{4}^{+}$(Section 4.3). Right panel: the 8-11.5 $\mu$ m region of the same sources as in the left panel showing the $\mathrm{C}-\mathrm{O}$ stretching mode of $\mathrm{CH}_{3} \mathrm{OH}$ (red) with the assumed local continuum (green) at the same column densities as in the left panel. Note that a scaling factor along the optical depth axis was applied for the spectra to fit in the panel.

(A color version of this figure is available in the online journal.)

Table 7

Intrinsic Integrated Band Strengths

\begin{tabular}{|c|c|c|c|}
\hline Molecule & Mode & $\begin{array}{c}A \\
\left(\mathrm{~cm} \text { molecule }^{-1}\right)\end{array}$ & Reference \\
\hline $\mathrm{H}_{2} \mathrm{O}$ & $3.0 \mu \mathrm{m}$ stretch & $2.0 \times 10^{-16}$ & Hagen et al. (1981) \\
\hline $\mathrm{NH}_{4}^{+}$ & $6.8 \mu \mathrm{m}$ deformation & $4.4 \times 10^{-17}$ & Schutte \& Khanna (2003) \\
\hline $\mathrm{CO}_{2}^{4}$ & $15.0 \mu \mathrm{m}$ bend & $1.1 \times 10^{-17}$ & Gerakines et al. (1995) \\
\hline $\mathrm{CH}_{3} \mathrm{OH}$ & $3.53 \mu \mathrm{m} \mathrm{C}-\mathrm{H}$ stretch & $5.6 \times 10^{-18}$ & Kerkhof et al. (1999) \\
\hline $\mathrm{CH}_{3} \mathrm{OH}$ & $9.7 \mu \mathrm{m} \mathrm{O}-\mathrm{H}$ stretch & $1.6 \times 10^{-17}$ & Kerkhof et al. (1999) \\
\hline $\mathrm{HCOOH}$ & $5.85 \mu \mathrm{m} \mathrm{C}=\mathrm{O}$ stretch & $6.7 \times 10^{-17}$ & Schutte et al. (1999) \\
\hline $\mathrm{HCOOH}$ & $7.25 \mu \mathrm{m}$ C-H deformation & $2.6 \times 10^{-18}$ & Schutte et al. (1999) \\
\hline $\mathrm{CH}_{4}$ & $7.68 \mu \mathrm{m}$ deformation & $7.3 \times 10^{-18}$ & Boogert et al. (1997) \\
\hline $\mathrm{NH}_{3}$ & $8.9 \mu \mathrm{m}$ umbrella & $1.3 \times 10^{-17}$ & Kerkhof et al. (1999) \\
\hline
\end{tabular}

$9.7 \mu \mathrm{m} \mathrm{C}-\mathrm{O}$ stretching modes are usually used for column density determinations (Boogert et al. 2008; Bottinelli et al. 2010). The $3.53 \mu \mathrm{m}$ band is preferred because it is free from interfering strong bands. Nevertheless it overlaps with the $3.47 \mu \mathrm{m}$ band of hydrocarbons (Allamandola et al. 1992; Brooke et al. 1996) or $\mathrm{NH}_{3}$ hydrates (Dartois \& d'Hendecourt 2001), as well as several photospheric lines. Thus, the most certain detections of solid $\mathrm{CH}_{3} \mathrm{OH}$ are toward background stars that show a distinct $3.53 \mu \mathrm{m}$ band. Figure 4 shows that this is certainly the case for two lines of sight: 2MASS J18171181-0814012 and 2MASS J18171366-0813188. The strongest detection (2MASS J18171181-0814012) was verified by observations in two different observing runs, using two different telluric standard stars. A good agreement is found for the strength of the $9.7 \mu \mathrm{m}$ band. Five additional lines of sight likely show solid $\mathrm{CH}_{3} \mathrm{OH}$ as well, although in these cases the $3.53 \mu \mathrm{m}$ feature is not as distinct from other fluctuations in the surrounding spectra (Figure 4). For all of these seven sources, the $\mathrm{CH}_{3} \mathrm{OH}$ abundance relative to $\mathrm{H}_{2} \mathrm{O}$ is in the $5 \%-12 \%$ range. A similar number of sources have upper limits of comparable magnitude, and thus the $\mathrm{CH}_{3} \mathrm{OH}$ abundance varies in different environments.
For the YSOs studied in Boogert et al. (2008), $\mathrm{HCOOH}$ is considered a detection only if the $7.25 \mu \mathrm{m} \mathrm{C}-\mathrm{H}$ deformation mode has been detected. This is not the case for any of the background stars. Based on the strength of the $\mathrm{C} 1$ component (Table 5), which may mostly be due to the $\mathrm{C}=\mathrm{O}$ stretch mode of $\mathrm{HCOOH}$, the abundance can in many lines of sight be constrained to less than or comparable to the typical detections toward YSOs of $2 \%-5 \%$ relative to $\mathrm{H}_{2} \mathrm{O}$, however (Table 6).

Finally, for $\mathrm{NH}_{3}$ and $\mathrm{CH}_{4}$ the $8.9 \mu \mathrm{m}$ umbrella and $7.68 \mu \mathrm{m}$ bending modes are used to determine column density upper limits. For $\mathrm{CH}_{4}$, the upper limits are sometimes comparable but rarely less than the detections of $\sim 4 \%$ toward YSOs (Öberg et al. 2008). For $\mathrm{NH}_{3}$, the upper limits are typically $\sim 20 \%$ relative to $\mathrm{H}_{2} \mathrm{O}$ (Table 6) and are thus not significant compared to the detections of 2\%-15\% toward YSOs (Bottinelli et al. 2010).

\subsection{Correlation Plots}

To facilitate the analysis of the observational parameters in many sight lines in many different environments (quiescent core and cloud media, low-mass YSOs, massive YSOs), a number of correlation plots are presented below. The values for the YSOs 

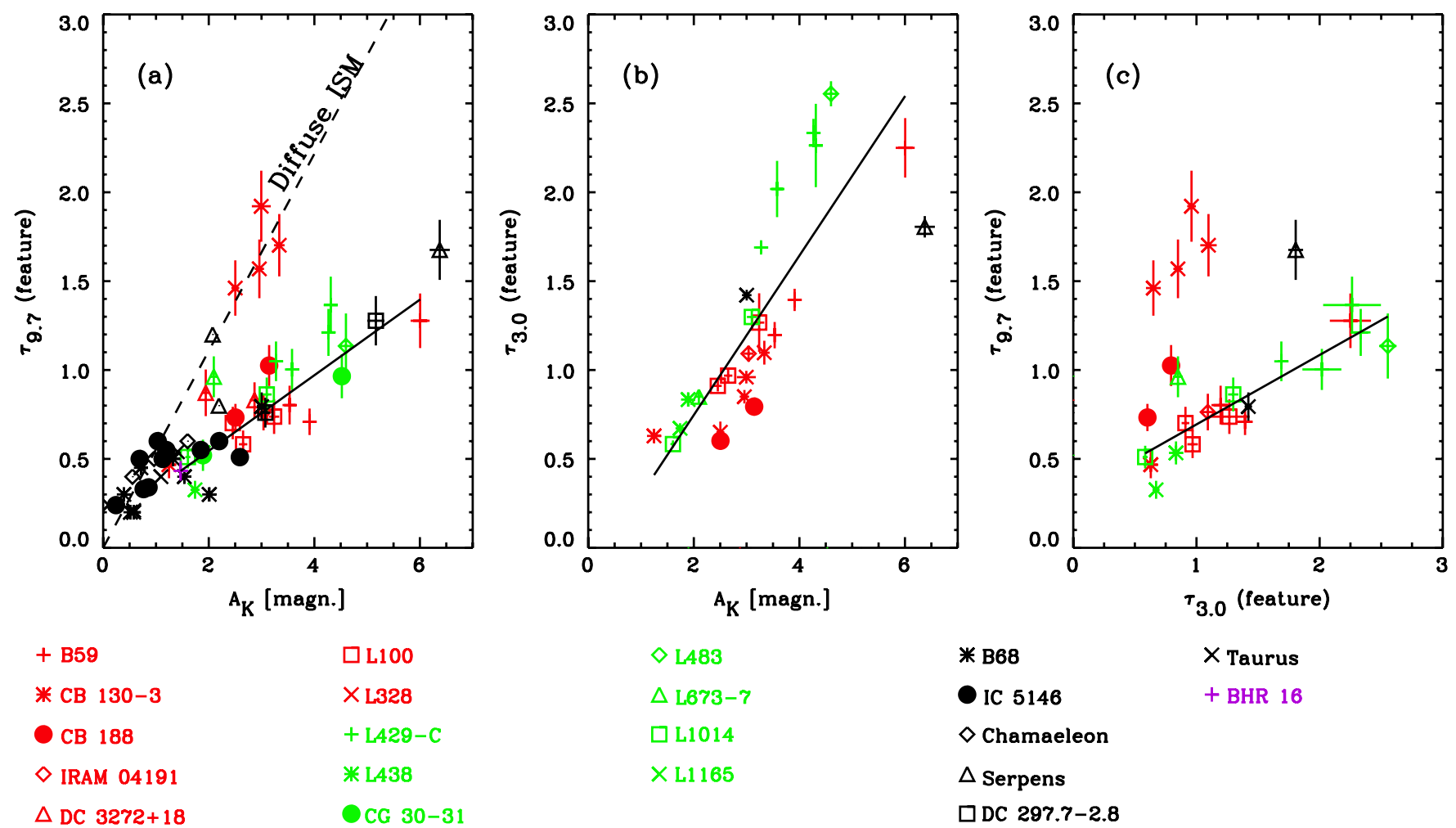

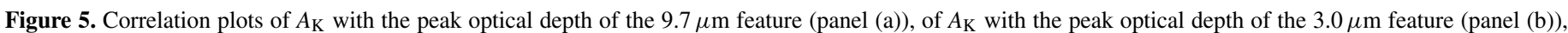

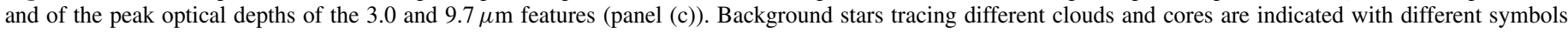

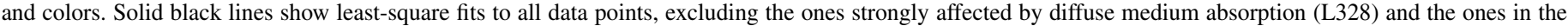

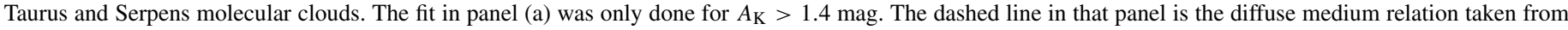
the literature (Whittet 2003), and the values for the B68, IC 5146, Chamaeleon, and most Serpens and Taurus sources were taken from Chiar et al. (2007).

(A color version of this figure is available in the online journal.)

were taken from Boogert et al. (2008) and Reach et al. (2009). Note that in plots involving $A_{\mathrm{K}}$ no YSOs are present because of the unknown shape of the intrinsic $K$-band continuum emission. Also, background stars with $\tau_{3.0}$ upper limits have been excluded from the plots.

$$
\text { 4.4.1. } \tau_{9.7} \text { versus } A_{K}
$$

The plot of the peak optical depth of the $9.7 \mu \mathrm{m}$ band of silicates against $K$-band continuum extinction $A_{\mathrm{K}}$ shows an interesting behavior (Figure 5(a)). While at low $A_{\mathrm{K}}$ the diffuse medium relation (Whittet 2003)

$$
\tau_{9.7}=0.554 \times A_{\mathrm{K}}
$$

is followed (assuming $A_{\mathrm{V}} / A_{\mathrm{K}}=8.8$ ), a less steep relation is observed at $A_{\mathrm{K}} \geqslant 1 \mathrm{mag}$ in dense sight lines. This was discovered by Chiar et al. (2007) in the IC 5146, B68 and B59 cores and Serpens and Taurus clouds, and here this behavior is extended in more lines of sight, at higher $K$-band extinctions. Some background stars, however, follow the diffuse medium correlation, most notably those behind L328, and, as noted by Chiar et al. (2007), one source behind Serpens (2MASS J18285266+0028242). Thus, in these lines of sight the extinction may well be dominated by diffuse rather than dense dust. Excluding these particular background stars, a polynomial was fitted to objects with $A_{\mathrm{K}}>1.4 \mathrm{mag}$ :

$$
\tau_{9.7}=(0.12 \pm 0.05)+(0.21 \pm 0.02) \times A_{\mathrm{K}} .
$$

It is worth noting that for some cores all background stars lie systematically above or below the fit, e.g., L429-c sources lie above it and B59 sources below it.

$$
\text { 4.4.2. } \tau_{3.0} \text { versus } A_{K}
$$

The peak optical depth of the $3.0 \mu \mathrm{m} \mathrm{H}_{2} \mathrm{O}$ ice band correlates strongly with $A_{\mathrm{K}}$ (Figure $5(\mathrm{~b})$ ). There is no indication of flattening. A linear fit to all data points yields

$$
\tau_{3.0}=(-0.15 \pm 0.13)+(0.45 \pm 0.05) \times A_{\mathrm{K}} .
$$

This relation implies a $\tau_{3.0}=0$ cutoff value of $A_{\mathrm{K}}=0.33 \pm$ 0.30 , which corresponds to $A_{\mathrm{V}}=2.9 \pm 2.6$ (assuming $A_{\mathrm{V}} / A_{\mathrm{K}}=$ 8.8). This is the so-called ice formation threshold, and it is comparable to the value observed for the Taurus Molecular Cloud $\left(A_{\mathrm{V}}=3.1 \pm 0.6\right.$; Chiar et al. 1995), but smaller than the $A_{\mathrm{V}}=10-15$ mag quoted by Tanaka et al. (1990) for Ophiuchus. The large uncertainty must reflect different ice formation thresholds in the different environments traced by the observations, or the presence of different amounts of ice-less diffuse dust along the line of sight. For example, all L328, B59, and CB 188 sources lie to the right of the fitted line. For L328, this most likely indicates a significant contribution of extinction in diffuse foreground clouds as indicated by the $\tau_{9.7}$ versus $\mathrm{A}_{\mathrm{K}}$ relation (Section 4.4.1). On the other hand, all L429-C sources lie to the left of the fit, indicating a low ice formation threshold.

$$
\text { 4.4.3. } \tau_{3.0} \text { versus } \tau_{9.7}
$$

Although the general relation of $\tau_{3.0}$ with $\tau_{9.7}$ is evident from panels (a) and (b) of Figure 5, it is plotted separately in panel (c). A linear fit to all points except those from L328 (Section 4.4.1) yields

$$
\tau_{9.7}=(0.36 \pm 0.09)+(0.36 \pm 0.06) \times \tau_{3.0} .
$$




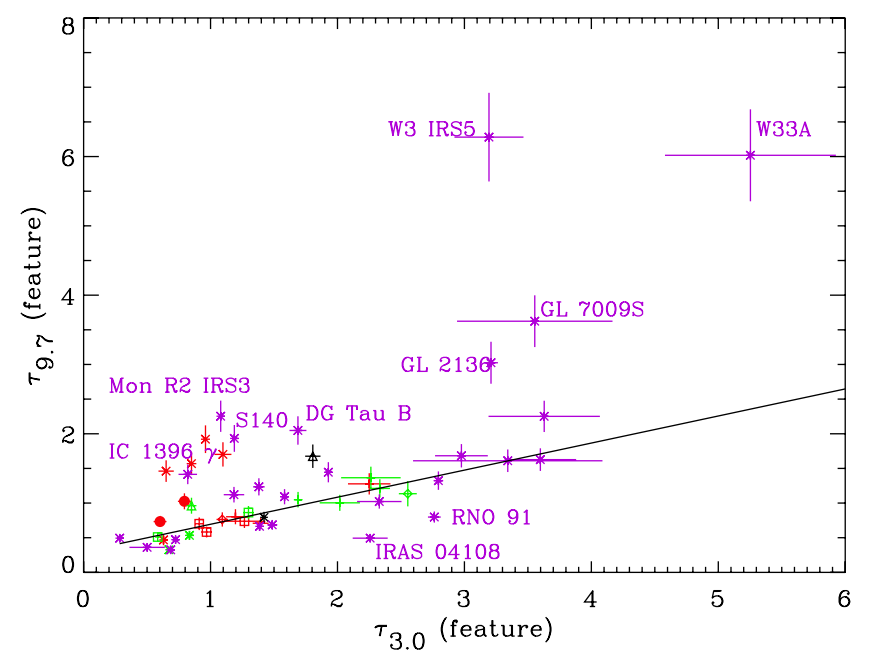

Figure 6. Peak optical depths of the 3.0 and $9.7 \mu \mathrm{m}$ absorption features for both background stars (in black, red, and green; see the legend of Figure 5) and YSOs (purple asterisks). Only YSOs with reliable $3 \mu \mathrm{m}$ spectra are plotted. The solid line represents the fit for background stars as derived in Figure 5(c). YSOs deviating more than $3 \sigma$ from the solid line are labeled, except for IC $1396 \alpha$ and B5 IRS1 (both above the solid line), and IRAS 03439, L1448 IRS1 (below the solid line), which are not labeled to avoid crowding in the plot.

(A color version of this figure is available in the online journal.)

For this particular relation, values for the YSOs are available from Boogert et al. (2008). They are displayed in Figure 6, where the linear fit from Equation. (6) above has been plotted. YSOs deviating more than $3 \sigma$ from the fit are labeled. YSOs lying below the line may have some of the silicate band filled in by emission, while sources above the line may have significant amounts of warm dust in which the $\mathrm{H}_{2} \mathrm{O}$ ices have evaporated. Interestingly, the latter are mostly the massive YSOs and YSOs that show a "red" $6.85 \mu \mathrm{m}$ band, indicative of thermally processed ices (Section 4.4.6).

\subsection{4. $6.0 \mu \mathrm{m}$ Band versus $N\left(\mathrm{H}_{2} \mathrm{O}\right)$}

Previous work by Keane et al. (2001) toward massive YSOs, Knez et al. (2005) toward background stars and Boogert et al.
(2008) toward low-mass YSOs has shown that the $6.0 \mu \mathrm{m}$ band can only partly be attributed to solid $\mathrm{H}_{2} \mathrm{O}$. Although $\mathrm{H}_{2} \mathrm{O}$ mixed with realistic concentrations of $\mathrm{CO}_{2}$ fits the long-wavelength side (component "C2" in Boogert et al. 2008) better than pure $\mathrm{H}_{2} \mathrm{O}$ (Knez et al. 2005; Öberg et al. 2007), it does not explain all absorption. This is the case for the background stars studied here as well. To quantify this, the integrated absorption remaining after subtraction of an amorphous, pure $\mathrm{H}_{2} \mathrm{O}$ laboratory ice at $T=10 \mathrm{~K}$ (Hudgins et al. 1993) is plotted against $N\left(\mathrm{H}_{2} \mathrm{O}\right)$, showing that the background stars are in line with the YSOs (Figure 7(a); Table 5). The scatter is large, however, which is reflected in a linear correlation coefficient of 0.79 . Some of this might be due to uncertainties in $N\left(\mathrm{H}_{2} \mathrm{O}\right)$, as many YSOs do not have reliable $3.0 \mu \mathrm{m}$ spectra available. Indeed, when only including sources with reliable $3.0 \mu \mathrm{m}$ spectra, the correlation coefficient increases to 0.85 . Another source of scatter may be the inaccuracy in the continuum determination, especially for the YSOs. Intrinsic abundance variations of the different carriers of the 5.2-6.4 $\mu \mathrm{m}$ "excess" absorption relative to $\mathrm{H}_{2} \mathrm{O}$ must cause some of the scatter as well (Figure 3).

\subsection{5. $6.85 \mu \mathrm{m}$ Band versus $N\left(\mathrm{H}_{2} \mathrm{O}\right)$}

The $6.85 \mu \mathrm{m}$ band is detected toward many background stars. Its integrated optical depth, calculated after subtracting the longwavelength wing of the $\mathrm{H}_{2} \mathrm{O}$ bending mode, is correlated with $N\left(\mathrm{H}_{2} \mathrm{O}\right)$ in Figure $7(\mathrm{~b})$, where the YSOs have been plotted as well. As for the $6.0 \mu \mathrm{m}$ band, the scatter is large. At a given value of $N\left(\mathrm{H}_{2} \mathrm{O}\right)$, the $6.85 \mu \mathrm{m}$ band depth varies by a factor of two. The linear correlation coefficient is 0.88 when including all background stars and YSOs. Within the scatter, there is no systematic difference in the strength of the $6.85 \mu \mathrm{m}$ band between YSOs and background stars.

\subsection{6. $\mathrm{C} 4 / \mathrm{C} 3$ Ratio versus $\mathrm{H}_{2} \mathrm{O}$ Abundance}

The 6.0 and $6.85 \mu \mathrm{m}$ bands were decomposed into components C1-C5 in Boogert et al. (2008). It was found that the C4/C3 component ratio varies significantly between YSOs. Sources with large $\mathrm{C} 4 / \mathrm{C} 3$ ratios tend to have low $\mathrm{H}_{2} \mathrm{O}$ ice abundances (represented by the $N\left(\mathrm{H}_{2} \mathrm{O}\right) / \tau_{9.7}$ ratio) and large degrees
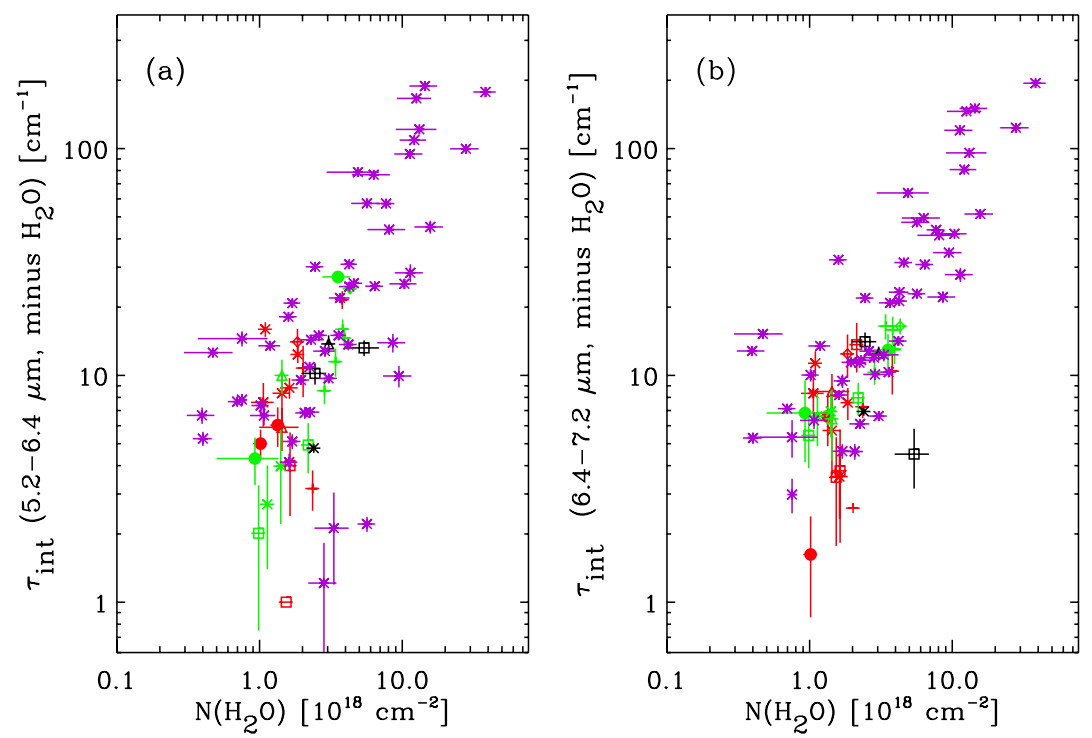

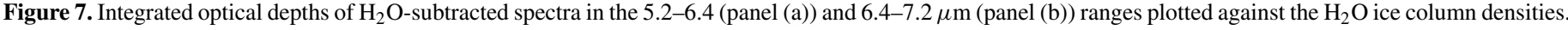

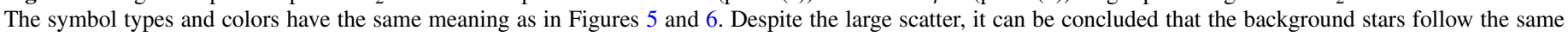
correlations as the YSOs (purple asterisks), i.e., the carriers of the absorption are already present in quiescent cloud material.

(A color version of this figure is available in the online journal.) 


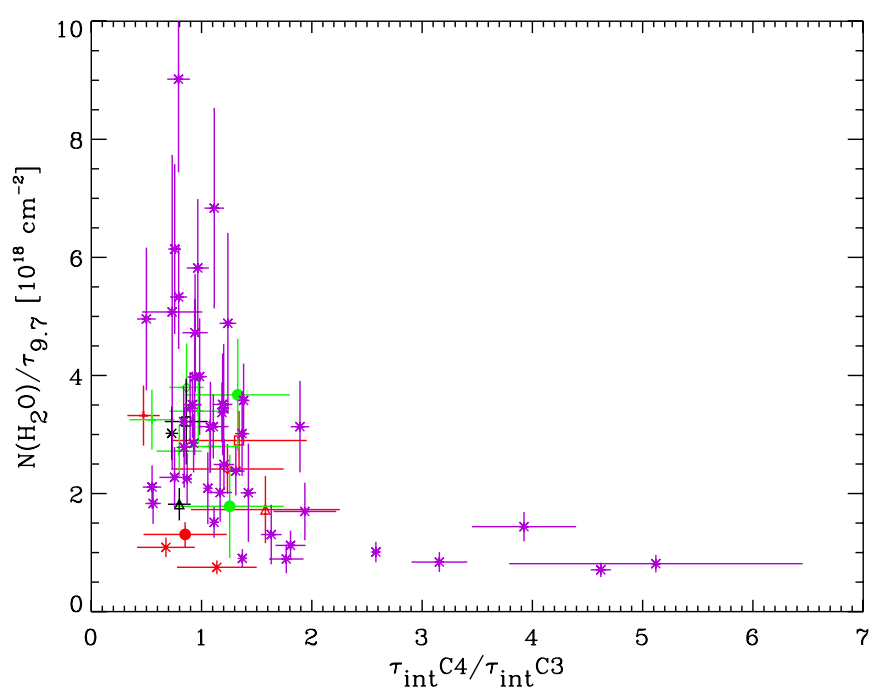

Figure 8. Ratio of integrated optical depths of the $\mathrm{C} 4$ and $\mathrm{C} 3$ components (long- and short-wavelength components of the $6.85 \mu \mathrm{m}$ band) plotted vs. the $\mathrm{H}_{2} \mathrm{O}$ ice column density normalized to the peak optical depth of the $9.7 \mu \mathrm{m}$ band (a measure of the $\mathrm{H}_{2} \mathrm{O}$ ice abundance). Only values with significance $>2 \sigma$ are plotted. Different colors and symbols refer to background stars behind different clouds and cores (see the legend of Figure 5). The values for the YSOs, indicated with purple asterisks, are taken from Boogert et al. (2008). While nearly all sources have $\mathrm{C} 4 / \mathrm{C} 3$ ratios near 1 , a number of YSOs have significantly larger ratios and at the same time low $\mathrm{H}_{2} \mathrm{O}$ abundances.

(A color version of this figure is available in the online journal.)

of ice crystallization and segregation (Keane et al. 2001; Boogert et al. 2008), thus indicating that the $\mathrm{C} 4 / \mathrm{C} 3$ ratio increases as a result of thermal processing. A similar decomposition was done for the background stars (Figure 8), and indeed they have low $\mathrm{C} 4 / \mathrm{C} 3$ ratios as expected for the cold, unprocessed sight lines that they trace. Nevertheless, some of the background stars (e.g., toward L328) have low $\mathrm{H}_{2} \mathrm{O}$ ice abundances, likely because of the presence of diffuse, ice-free, foreground clouds (Section 4.4.1) rather than because of the effects of ice sublimation as for YSOs.

\section{DISCUSSION}

\subsection{Ice Abundances}

The infrared spectra of ices and dust toward background stars presented here provide a baseline for the astrochemical evolution of YSOs. It is shown that the strength and shape of the 3.0,6.0,6.85, and $15 \mu \mathrm{m}$ ice bands are similar for the quiescent material in isolated cores traced by background stars compared to most previously studied YSOs. Correspondingly, the relative abundances of the ices with secure $\left(\mathrm{CO}_{2}, \mathrm{CH}_{3} \mathrm{OH}\right)$ and less secure identifications $\left(\mathrm{NH}_{4}^{+}\right)$are similar to most YSOs. Species responsible for the $6.0 \mu \mathrm{m}$ band, besides $\mathrm{H}_{2} \mathrm{O}$ (probably $\mathrm{HCOOH}, \mathrm{H}_{2} \mathrm{CO}$, and $\mathrm{NH}_{3}$ ), must have abundances similar to YSOs as well. This confirms earlier work on smaller samples (Knez et al. 2005; Bergin et al. 2005), and it firmly establishes that most ices are formed early on in the molecular cloud evolution, and are largely unaltered during the further evolution of the region (e.g., the star formation process) until the YSO thermally processes the ices in the envelope.

The two background stars with the most secure $\mathrm{CH}_{3} \mathrm{OH}$ detections (2MASS J18171181-0814012 and 2MASS J181713660813188 ), at $\sim 11 \%$ with respect to $\mathrm{H}_{2} \mathrm{O}$, are both located behind the core L429-C. These lines of sight show some of the deepest ice and dust features in the sample, which may have facilitated the detection of the weak $3.53 \mu \mathrm{m} \mathrm{CH} \mathrm{CH}_{3} \mathrm{OH}$ feature. Indeed, many other background stars in the sample have upper limits or tentative detections at abundances not much less $(6 \%-10 \%)$ than the detections. Some, e.g., 2MASS J04393886+2611266 in the Taurus Molecular Cloud, have tighter upper limits of 1\%-3\% (Chiar et al. 1996; Table 6), however. Large $\mathrm{CH}_{3} \mathrm{OH}$ abundance variations are also observed toward YSOs (Dartois et al. 1999; Pontoppidan et al. 2003a; Boogert et al. 2008).

The presence of solid $\mathrm{CH}_{3} \mathrm{OH}$ in quiescent clouds is not unexpected, following theoretical studies (Tielens \& Allamandola 1987; Keane 2001), laboratory studies (Watanabe \& Kouchi 2002; Fuchs et al. 2009), and time-dependent (Monte Carlo) simulations (Cuppen et al. 2009). While the laboratory experiments have not yet been fully translated into kinetic parameters, in a general sense, the theoretical studies and kinetic Monte Carlo simulations do show that the $\mathrm{CH}_{3} \mathrm{OH} / \mathrm{CO}$ ratio is very sensitive to the dust temperature and to the ratio of atomic $\mathrm{H}$ to $\mathrm{CO}$ in the accreting gas (Keane 2001; Cuppen et al. 2009). In this model, an $\mathrm{H}_{2} \mathrm{O}$-rich mantle forms first, because of its higher sublimation temperature, and at $T<17 \mathrm{~K}$ a CO mantle forms on top of that. Accreting $\mathrm{H}$ atoms then form $\mathrm{H}_{2} \mathrm{CO}$ and $\mathrm{CH}_{3} \mathrm{OH}$. High $\mathrm{CH}_{3} \mathrm{OH} / \mathrm{CO}$ ratios are indicative of high $\mathrm{H} / \mathrm{CO}$ ratios of the accreting gas (due to relatively low densities or high local cosmic-ray fluxes that destroy $\mathrm{H}_{2}$ ). Alternatively, low $\mathrm{CH}_{3} \mathrm{OH} / \mathrm{CO}$ ratios may reflect relatively elevated dust temperatures ( $T>15 \mathrm{~K}$; Cuppen et al. 2009). Thus, local physical conditions are a dominating factor in the formation of $\mathrm{CH}_{3} \mathrm{OH}$, and this may explain the large observed $\mathrm{CH}_{3} \mathrm{OH}$ abundance variations in different lines of sight.

\subsection{Processing of the Ices}

Ice evolution is observed in the envelopes of some YSOs, and it appears that this is mostly due to the effects of heating: apolar CO-rich ices sublimate and $\mathrm{H}_{2} \mathrm{O}$-rich ices crystallize (Smith et al. 1989; Gerakines et al. 1999; Boogert et al. 2000, 2008; Pontoppidan et al. 2008). The carrier of the $6.85 \mu \mathrm{m}$ band is affected by heating as well: it shifts to longer wavelengths (Keane et al. 2001; Boogert et al. 2008). Toward background stars, none of these effects are observed. Figure 8 shows that all background stars with high-quality spectra have $6.85 \mu \mathrm{m}$ bands peaking at short wavelengths (low $\mathrm{C} 4 / \mathrm{C} 3$ ratios). The observed $3.0 \mu \mathrm{m}$ bands (Figure 9) are characteristic of low-temperature, amorphous ices. Crystalline ices would peak near $3.1 \mu \mathrm{m}$ (Smith et al. 1989).

Finally, an absorption feature in the 5-8 $\mu \mathrm{m}$ region referred to as C5 in Boogert et al. (2008; see also Gibb \& Whittet 2002) has not been observed toward background stars (Table 5). Although continuum reliability is generally a problem for this shallow feature, detections $(>3 \sigma)$ were claimed in $\sim 30 \%$ of the low-mass YSOs and $\sim 50 \%$ of the massive YSOs. The feature could be due to the formation of new molecules by energetic processing of the ices, possibly hydrocarbons (Greenberg et al. 1995) or ions (Schutte \& Khanna 2003). Its absence toward background stars is consistent with an origin in ices processed in the YSO environment, and not by external cosmic rays.

\subsection{Ices, Silicates, and the Extinction Curve}

Observations show that the properties of dust in diffuse and dense clouds are different. In contrast to diffuse clouds, the mid-infrared extinction in the dense cores studied here $\left(A_{\mathrm{K}}>1 \mathrm{mag}\right)$ stays high: at $25 \mu \mathrm{m}$ it is still half of that at $2 \mu \mathrm{m}$ (Figure 2(b); Chapman et al. 2009; McClure 2009). This 


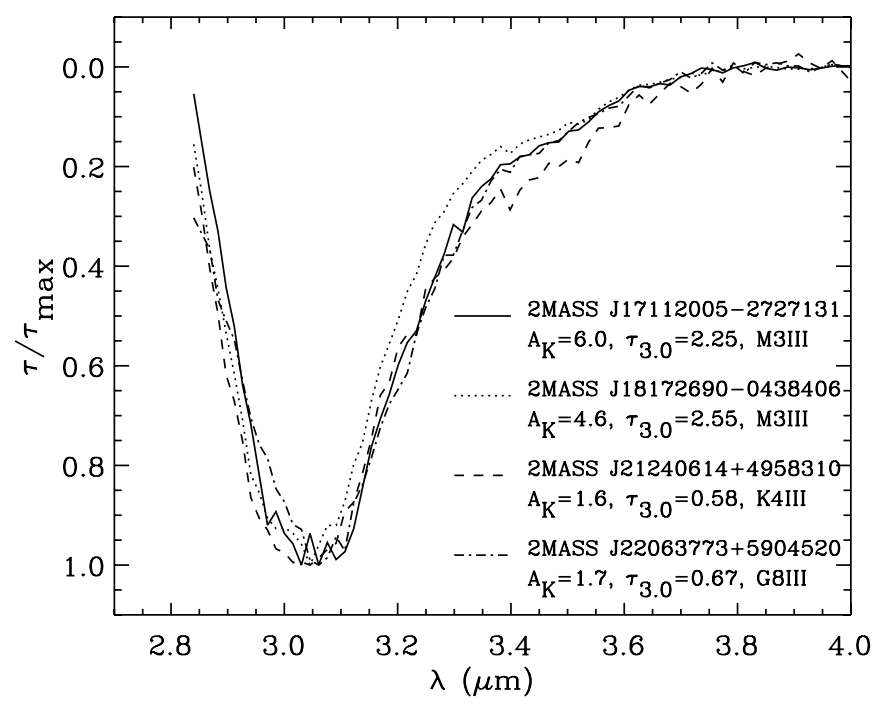

Figure 9. Profiles of the $3 \mu \mathrm{m}$ ice bands observed toward two background stars with the highest (solid and dotted lines), and two background stars with the lowest (dashed and dash-dotted lines) extinctions and peak optical depths in the sample. The spectra are smoothed to a resolving power of 100 . The profiles are very similar, indicating a lack of grain growth.

is most likely caused by grain growth. Indeed, the calculated $R_{\mathrm{V}}=5.5$ ("case B") extinction curve from Weingartner \& Draine (2001) is closer to the observations than their $R_{\mathrm{V}}=$ 3.1 "case A" model (Figure 2(c); Chapman et al. 2009; McClure 2009). Nevertheless, even this extreme case of $R_{\mathrm{V}}=5.5$ is systematically too shallow at wavelengths above $5 \mu \mathrm{m}$, while the silicate features are too deep. The present work shows that the contribution of ices, which are not included in the Weingartner $\&$ Draine (2001) models, to the extinction is not very large: $\sim 25 \%$ in the $11-17 \mu \mathrm{m}$ wavelength region. Instead, a model with larger grain sizes is needed. Such a model would also need to take into account the observed weakness of the $9.7 \mu \mathrm{m}$ band of silicates (Figures 2(c) and 5(a)).

Despite the need for larger grains in dense clouds, compared to diffuse clouds, there is no evidence for progressive grain growth in dense clouds for $A_{\mathrm{K}}>1 \mathrm{mag}$. The empirical extinction curve (Figure 2) satisfactorily fits most background stars in the sample, for the range of observed $A_{\mathrm{K}}$ values of 1.3-6 mag. The same conclusion can be drawn from Figure 1 in McClure (2009). Also, the $3.0 \mu \mathrm{m}$ band profiles are very similar over this $A_{\mathrm{K}}$ range (Figure 9), while a broadening and shift to longer wavelengths would be expected if grain growth occurred. Similarly, the observed linear relation of the $3.0 \mu \mathrm{m}$ band with dust column density indicators such as the $9.7 \mu \mathrm{m}$ band of silicates and $A_{\mathrm{K}}$ (Figure 5) indicates a lack of progressive grain growth, since larger grains produce $3.0 \mu \mathrm{m}$ bands with smaller peak optical depths.

Recent work shows that the silicate band profiles are inconsistent with grain growth beyond $1 \mu \mathrm{m}$ (van Breemen et al. 2011), similar to what is found for the $3.0 \mu \mathrm{m}$ ice band (Smith et al. 1989). It is thus unlikely that grain growth can fully explain the shallower $A_{\mathrm{K}}$ versus $\tau_{9.7}$ relation in the dense medium compared to the diffuse medium (Figure 5(a)). Different dust compositions may play a role as well. The silicate bands in dense clouds show a short-wavelength wing that could be due to a larger pyroxene/ olivine abundance ratio (Section 4.1). For a more thorough investigation of the $A_{\mathrm{K}}$ versus $\tau_{9.7}$ relation one is referred to van Breemen et al. (2011).

\section{CONCLUSIONS AND FUTURE WORK}

The analysis of 2-25 $\mu \mathrm{m}$ photometry and spectra of 31 stars tracing the quiescent medium in 16 isolated dense cores yields the following conclusions.

1. An empirical "high-resolution" extinction curve derived from one of the background stars confirms extinction curves derived from broadband photometry in high density clouds (Chapman et al. 2009). The curve remains remarkably flat above $10 \mu \mathrm{m}$, such that at $25 \mu \mathrm{m}$ the extinction is still half that at $2.2 \mu \mathrm{m}$. The main caveat is that it is assumed the nearinfrared curve is the same as that derived by Indebetouw et al. (2005).

2. The empirical extinction curve, in combination with model spectra of giants, and absorption spectra of $\mathrm{H}_{2} \mathrm{O}$ ice and silicates is able to fit the observed data of most background stars well. No systematic deviations are seen as a function of core or $A_{\mathrm{K}}$ values (noting that the sample includes only high extinction lines of sight with $A_{\mathrm{K}} \gtrsim 1.5 \mathrm{mag}$ ).

3. Model fits of the attenuated stellar continuum are used to isolate the ice and dust absorption features. The $9.7 \mu \mathrm{m}$ band of silicates shows a shallower relation with $A_{\mathrm{K}}$ compared to the diffuse ISM, thus confirming the results of Chiar et al. (2007) in many more lines of sight. Some background stars, e.g., those behind the L328 core, follow the diffuse medium relation, however.

4. The $3.0 \mu \mathrm{m}$ band of $\mathrm{H}_{2} \mathrm{O}$ ice is detected in all observed sources. A strong correlation is observed between its peak optical depth and $A_{\mathrm{K}}$. Its shape is independent of $A_{\mathrm{K}}$, indicating a lack of grain growth at high $A_{\mathrm{K}}$ values. This may imply that the shallower relation of $\tau_{9.7}$ with $A_{\mathrm{K}}$ in the dense ISM compared to the diffuse ISM is not caused by grain growth. Possibly the dust composition is different, as also suggested by the broader $9.7 \mu \mathrm{m}$ band in dense sight lines (both YSOs and background stars).

5. The 6.0 and $6.85 \mu \mathrm{m}$ absorption bands are detected in most background stars. As for YSOs, the $6.0 \mu \mathrm{m}$ band is only partially explained by the bending mode of $\mathrm{H}_{2} \mathrm{O}$ ice. Additional species, such as $\mathrm{HCOOH}, \mathrm{H}_{2} \mathrm{CO}, \mathrm{CH}_{3} \mathrm{OH}$, and $\mathrm{NH}_{3}$ must be responsible for this. Together with the consistent presence of the $6.85 \mu \mathrm{m}$ band, which may be due to $\mathrm{NH}_{4}^{+}$, this shows that there is little difference between the ices in quiescent core regions and those surrounding most YSOs. Most ices are apparently formed in the quiescent cloud phase.

6. The discovery of solid $\mathrm{CH}_{3} \mathrm{OH}$ in the $L$-band spectra of several background stars strengthens the previous point. It confirms recent models by Cuppen et al. (2009) that $\mathrm{CH}_{3} \mathrm{OH}$ is efficiently formed on the grains in low-temperature $(T<$ $15 \mathrm{~K}$ ) clouds. Tight upper limits in previously studied sight lines indicate that the ice composition varies in different environments at the onset of star formation, however. For a proper comparison with the models, observed $\mathrm{CH}_{3} \mathrm{OH} /$ $\mathrm{CO}$ ratios are needed, i.e., more observations of solid $\mathrm{CO}$ toward background stars.

7. Signatures that are assigned to processing of the ices surrounding YSOs, such as profile changes in the $3 \mu \mathrm{m}$ ice band, the shifted $6.85 \mu \mathrm{m}$ band (i.e., a large C4/C3 ratio) and the presence of the broad $\mathrm{C} 5$ components, are not found toward the background stars.

The present work studies ices and the extinction curve in the quiescent medium in isolated dense cores. YSOs in these cores are not well studied. On the other hand, the ices are well studied 
toward YSOs in large clouds (Taurus, Serpens, Oph, Perseus; Boogert et al. 2008; Pontoppidan et al. 2008; Öberg et al. 2008; Bottinelli et al. 2010), but the background star samples are small (Knez et al. 2005). Thus, future studies will need to compare YSOs and background stars in the same environments to address the origin of the observed variations of the $\mathrm{CH}_{3} \mathrm{OH}$ abundance and the strength of the 6.0 and $6.8 \mu \mathrm{m}$ ice bands (Figures 3 and 7). Furthermore, while there is now much observational evidence that the dust properties in the dense and diffuse medium are quite different, the cause of this is not understood, warranting further theoretical and perhaps laboratory investigations.

We thank the anonymous referee for comments that helped with improving the presentation and conclusions of this work. T.L.H. acknowledges support for this work provided by NASA through contract 1316720 issued by JPL/Caltech. A.M.C. acknowledges support from the NASA Astrobiology Institute (grant NNA09DA80A) and the IPAC Visiting Graduate Student Fellowship Program. This publication makes use of data products from the Two Micron All Sky Survey, which is a joint project of the University of Massachusetts and the Infrared Processing and Analysis Center/California Institute of Technology, funded by the National Aeronautics and Space Administration and the National Science Foundation.

\section{REFERENCES}

Allamandola, L. J., Sandford, S. A., Tielens, A. G. G. M., \& Herbst, T. M. 1992, ApJ, 399, 134

Beichman, C. A., Myers, P. C., Emerson, J. P., Harris, S., Mathieu, R., Benson, P. J., \& Jennings, R. E. 1986, ApJ, 307, 337

Bergin, E. A., Melnick, G. J., Gerakines, P. A., Neufeld, D. A., \& Whittet, D. C. B. 2005, ApJ, 627, L33

Bernstein, M. P., Dworkin, J. P., Sandford, S. A., Cooper, G. W., \& Allamandola, L. J. 2002, Nature, 416, 401

Bohren, C. F., \& Huffman, D. R. 1983, Absorption and Scattering of Light by Small Particles (New York: Wiley)

Boogert, A. C. A., Blake, G. A., \& Tielens, A. G. G. M. 2002, ApJ, 577, 271

Boogert, A. C. A., \& Ehrenfreund, P. 2004, in ASP Conf. Ser. 309, Astrophysics of Dust, ed. A. N. Witt, G. C. Clayton, \& B. T. Draine (San Francisco, CA: ASP), 547

Boogert, A. C. A., Schutte, W. A., Helmich, F. P., Tielens, A. G. G. M., \& Wooden, D. H. 1997, A\&A, 317, 929

Boogert, A. C. A., et al. 2000, A\&A, 353, 349

Boogert, A. C. A., et al. 2008, ApJ, 678, 985

Bottinelli, S., et al. 2010, ApJ, 718, 1100

Brooke, T. Y., Sellgren, K., \& Smith, R. G. 1996, ApJ, 459, 209

Chapman, N. L., Mundy, L. G., Lai, S.-P., \& Evans, N. J. 2009, ApJ, 690, 496

Chiar, J. E., Adamson, A. J., Kerr, T. H., \& Whittet, D. C. B. 1995, ApJ, 455, 234

Chiar, J. E., Adamson, A. J., \& Whittet, D. C. B. 1996, ApJ, 472, 665

Chiar, J. E., et al. 2007, ApJ, 666, L73

Chiar, J. E., et al. 2011, ApJ, in press

Cuppen, H. M., van Dishoeck, E. F., Herbst, E., \& Tielens, A. G. G. M. 2009, A\&A, 508, 275

Dartois, E., \& d'Hendecourt, L. 2001, A\&A, 365, 144

Dartois, E., Schutte, W., Geballe, T. R., Demyk, K., Ehrenfreund, P., \& d'Hendecourt, L. 1999, A\&A, 342, L32

Decin, L., Morris, P. W., Appleton, P. N., Charmandaris, V., Armus, L., \& Houck, J. R. 2004, ApJS, 154, 408

Dorschner, J., Begemann, B., Henning, T., Jaeger, C., \& Mutschke, H. 1995, A\&A, 300, 503
Evans, N. J., II 1999, ARA\&A, 37, 311

Evans, N. J., II, et al. 2003, PASP, 115, 965

Evans, N. J., II, et al. 2007, Final Delivery of Data from the c2d Legacy Project: IRAC and MIPS (Pasadena, CA: SSC), http://ssc.spitzer.caltech.edu/legacy/

Fuchs, G. W., Cuppen, H. M., Ioppolo, S., Romanzin, C., Bisschop, S. E., Andersson, S., van Dishoeck, E. F., \& Linnartz, H. 2009, A\&A, 505, 629

Gerakines, P. A., Schutte, W. A., Greenberg, J. M., \& van Dishoeck, E. F. 1995 , A\&A, 296, 810

Gerakines, P. A., et al. 1999, ApJ, 522, 357

Gibb, E. L., \& Whittet, D. C. B. 2002, ApJ, 566, L113

Gibb, E. L., Whittet, D. C. B., Boogert, A. C. A., \& Tielens, A. G. G. M. 2004, ApJS, 151, 35

Greenberg, J. M., Li, A., Mendoza-Gomez, C. X., Schutte, W. A., Gerakines, P. A., \& de Groot, M. 1995, ApJ, 455, L177

Hagen, W., Tielens, A. G. G. M., \& Greenberg, J. M. 1981, Chem. Phys., 56, 367

Hudgins, D. M., Sandford, S. A., Allamandola, L. J., \& Tielens, A. G. G. M 1993, ApJS, 86, 713

Indebetouw, R., et al. 2005, ApJ, 619, 931

Kane, S. R., \& Sahu, K. C. 2003, ApJ, 582, 743

Keane, J. V. 2001, PhD thesis, Univ. Groningen

Keane, J. V., Tielens, A. G. G. M., Boogert, A. C. A., Schutte, W. A., \& Whittet, D. C. B. 2001 , A\&A, 376, 254

Kemper, F., Vriend, W. J., \& Tielens, A. G. G. M. 2004, ApJ, 609, 826

Kerkhof, O., Schutte, W. A., \& Ehrenfreund, P. 1999, A\&A, 346, 990

Knez, C., et al. 2005, ApJ, 635, L145

Lada, C. J., Alves, J., \& Lada, E. A. 1999, in Proc. 3rd Cologne-Zermatt Symposium, The Physics and Chemistry of the Interstellar Medium, Vol. 161, ed. V. Ossenkopf, J. Stutzki, \& G. Winnewisser (Herdecke: GCA-Verlag)

McClure, M. 2009, ApJ, 693, L81

McLean, I. S., et al. 1998, Proc. SPIE, 3354, 566

Myers, P. C., \& Benson, P. J. 1983, ApJ, 266, 309

Öberg, K. I., Boogert, A. C. A., Pontoppidan, K. M., Blake, G. A., Evans, N. J., Lahuis, F., \& van Dishoeck, E. F. 2008, ApJ, 678, 1032

Öberg, K. I., Fraser, H. J., Boogert, A. C. A., Bisschop, S. E., Fuchs, G. W., van Dishoeck, E. F., \& Linnartz, H. 2007, A\&A, 462, 1187

Pontoppidan, K. M., Dartois, E., van Dishoeck, E. F., Thi, W.-F., \& d'Hendecourt, L. 2003a, A\&A, 404, L17

Pontoppidan, K. M., et al. 2003b, A\&A, 408, 981

Pontoppidan, K. M., et al. 2008, ApJ, 678, 1005

Reach, W. T., et al. 2009, ApJ, 690, 683

Schutte, W. A., Allamandola, L. J., \& Sandford, S. A. 1993, Icarus, 104, 118

Schutte, W. A., \& Khanna, R. K. 2003, A\&A, 398, 1049

Schutte, W. A., et al. 1999, A\&A, 343, 966

Shu, F. H., Adams, F. C., \& Lizano, S. 1987, ARA\&A, 25, 23

Skrutskie, M. F., et al. 2006, AJ, 131, 1163

Smith, R. G., Sellgren, K., \& Tokunaga, A. T. 1989, ApJ, 344, 413

Tanaka, M., Sato, S., Nagata, T., \& Yamamoto, T. 1990, ApJ, 352, 724

Tielens, A. G. G. M., \& Allamandola, L. J. 1987, Interstellar Processes, Vol. 134 (Dordrecht: Reidel), 397

Tielens, A. G. G. M., \& Hagen, W. 1982, A\&A, 114, 245

Tielens, A. G. G. M., Tokunaga, A. T., Geballe, T. R., \& Baas, F. 1991, ApJ, 381,181

van Breemen, J. M., et al. 2011, A\&A, 526, A152

Watanabe, N., \& Kouchi, A. 2002, ApJ, 571, L173

Weingartner, J. C., \& Draine, B. T. 2001, ApJ, 548, 296

Whittet, D. C. B. (ed.) 2003, Dust in the Galactic Environment (Series in Astronomy and Astrophysics; 2nd ed.; Bristol: Institute of Physics Publishing)

Whittet, D. C. B., Bode, M. F., Baines, D. W. T., Longmore, A. J., \& Evans, A. 1983, Nature, 303, 218

Whittet, D. C. B., Cook, A. M., Chiar, J. E., Pendleton, Y. J., Shenoy, S. S., \& Gerakines, P. A. 2009, ApJ, 695, 94

Whittet, D. C. B., Pendleton, Y. J., Gibb, E. L., Boogert, A. C. A., Chiar, J. E., \& Nummelin, A. 2001, ApJ, 550, 793

Whittet, D. C. B., et al. 1998, ApJ, 498, L159

Young, C. H., et al. 2004, ApJS, 154, 396

Zasowski, G., Kemper, F., Watson, D. M., Furlan, E., Bohac, C. J., Hull, C., \& Green, J. D. 2009, ApJ, 694, 459 